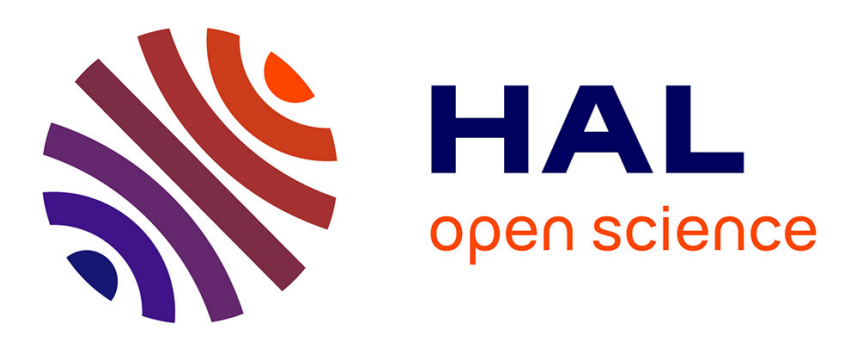

\title{
Analytic Derivative Calculation of Electronic g-Tensors based on Multireference Configuration Interaction Wavefunctions
}

Frank Neese

\section{- To cite this version: \\ Frank Neese. Analytic Derivative Calculation of Electronic g-Tensors based on Multireference Configuration Interaction Wavefunctions. Molecular Physics, 2008, 105 (19-22), pp.2507-2514. 10.1080/00268970701549389 . hal-00513126}

\author{
HAL Id: hal-00513126 \\ https://hal.science/hal-00513126
}

Submitted on 1 Sep 2010

HAL is a multi-disciplinary open access archive for the deposit and dissemination of scientific research documents, whether they are published or not. The documents may come from teaching and research institutions in France or abroad, or from public or private research centers.
L'archive ouverte pluridisciplinaire HAL, est destinée au dépôt et à la diffusion de documents scientifiques de niveau recherche, publiés ou non, émanant des établissements d'enseignement et de recherche français ou étrangers, des laboratoires publics ou privés. 


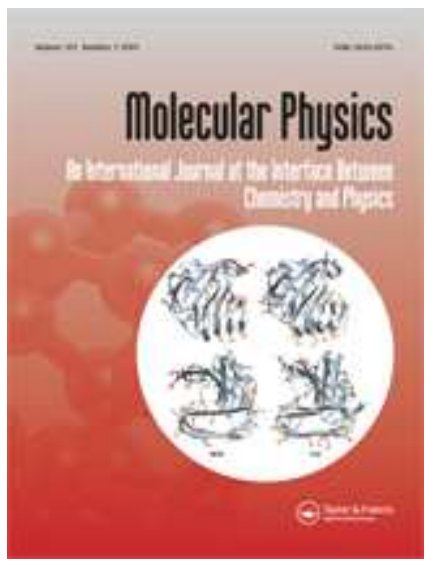

\section{Analytic Derivative Calculation of Electronic g-Tensors based on Multireference Configuration Interaction Wavefunctions}

\begin{tabular}{|r|l|}
\hline Journal: & Molecular Physics \\
\hline Manuscript ID: & TMPH-2007-0115.R1 \\
\hline Manuscript Type: & Full Paper \\
\hline $\begin{array}{r}\text { Date Submitted by the } \\
\text { Author: }\end{array}$ & 25-Jun-2007 \\
\hline Complete List of Authors: & $\begin{array}{l}\text { Neese, Frank; Bonn University, Institute for Physical and } \\
\text { Theoretical Chemistry }\end{array}$ \\
\hline Keywords: & EPR, g-tensor, MRCI, Linear response, Analytic derivatives \\
\hline \multicolumn{2}{|l}{} \\
\hline
\end{tabular}

\section{scholaronE \\ Manuscript Central}




\section{Analytic Derivative Calculation of Electronic g-Tensors based on Multireference Configuration Interaction Wavefunctions}

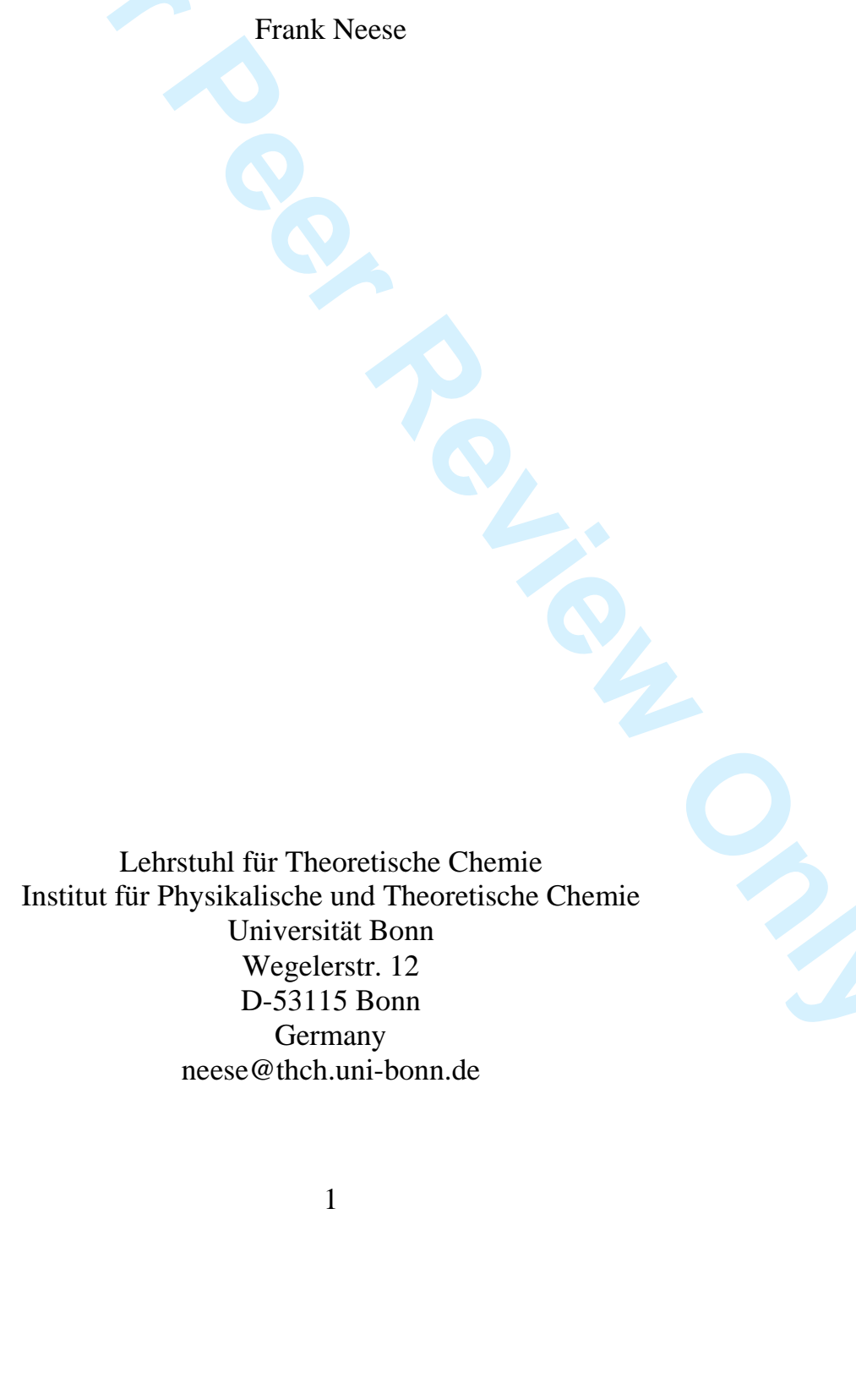




\begin{abstract}
A computer program has been written to perform analytic derivative calculations of magnetic response properties based on uncontracted multireference configuration interaction (MRCI) wavefunctions. At this point orbital relaxation is neglected in this treatment and the basis functions are assumed to be independent of the perturbation. It is therefore equivalent to an untruncated sum-over-states (SOS) approach to the g-tensor. Hence, the treatment is more rigorous than all previous MRCI g-tensor calculations that have used truncated SOS formulations. In the present calculations, MR-CI calculations were performed on top of full valence complete-active space self-consistent field (CASSCF) reference wavefunctions without truncation of the reference or correlation spaces. Results are reported for two types of spin-orbit coupling operators, a simple effective nuclear charge approximation and the spinorbit mean field (SOMF) approximation. The effects of orbital relaxation are discussed. The values obtained can be used in future calibration studies.
\end{abstract} Formatiert: Englisch 
1

2

3

4

5

6

7

8

9

\section{Introduction}

The theory of the g-tensor, the central property measured in electron paramagnetic resonance (EPR) experiments, has undergone a rapid development in recent years. In particular, methods based on density functional theory (DFT) have become very popular and are now widely applied in the interpretation of experiments (for reviews see [1-3]). However, one difficulty has been to find highly accurate data for calibration purposes since the g-values measured for small molecules in matrix isolation or the gas phase show a large scatter among different investigations (see for example the data assembled in ref [4]). Thus, there is a need for highly accurate reference data to gauge the quality of approximate approaches. In the $a b$ initio field, g-tensors have been previously calculated by analytic derivative UHF theory,[5] generalized Hartree-Fock theory[6] and sum-over-states (SOS) based formulations for restricted-openshell Hartree-Fock (ROHF), multireference configuration-interaction (MRCI) [7-19] as well as multireference perturbation $[18,20-23]$ approaches. In addition, a linear response theory (LRT) for g-tensors based on the complete active space self-consistent field (CASSCF) method has been developed and applied.[24-27]

In this paper, an analytic derivative (linear response) method for the calculation of electronic g-tensors based on MRCI wavefunctions is reported. The method is approximate in thus far, as that orbital relaxation contributions and the dependence of the basis set on the perturbation are neglected. The present development is considered to be important since MRCI treatments are the most general and robust approach to electronic structure theory as they treat both static and dynamic correlation contributions in a balanced way.

\section{Theory}

The theory of analytic first- and second-derivatives for MR-CI wavefunctions is well known and will only be briefly described in order to document what was actually programmed. We basically follow Kallay and Gauss who have recently described general methods to obtain
Feldfunktion geändert

Feldfunktion geändert Feldfunktion geändert

Feldfunktion geändert

Feldfunktion geändert

Feldfunktion geändert 
analytic first- and second-derivatives for arbitrary CI and coupled-cluster (CC) wavefunctions.[28,29] The former case is considerably simpler since the MRCI energy is stationary with respect to variations in the CI coefficients. The MRCI wavefunction (assumed to be normalized) is given by:

$$
\Psi_{M R C I}=\sum_{I} C_{I}^{(0)} \Phi_{I}
$$

The variational CI coefficients $C_{I}^{(0)}$ (the superscript (0) denotes the unperturbed situation) have been obtained by diagonalizing the Born-Oppenheimer Hamiltonian:

$$
\hat{H}^{(0)}=\hat{V}_{N N}+\sum_{p, q} h_{p q} E_{p q}^{+}+\frac{1}{2} \sum_{p, q, r, s}(p q \mid r s)\left[E_{p q}^{+} E_{r s}^{+}-\delta_{q r} E_{p s}^{+}\right]
$$

in the space of some configuration-state functions (CSFs) $\{\Phi\}$ of the desired spin- and spacesymmetry (e.g. by solving $\left.\left(\mathbf{H}^{(0)}-\mathbf{1} E_{M R C I}\right) \mathbf{C}^{(0)}=0\right) . \hat{V}_{N N}$ is the nuclear repulsion energy, $h_{p q}$ the matrix elements of the one-electron operator and $(p q \mid r s)$ are the two-electron repulsion integrals in charge cloud notation. The orbital replacement operators are defined by:

$$
E_{p q}^{ \pm}=a_{p \alpha}^{+} a_{q \alpha} \pm a_{p \beta}^{+} a_{q \beta}
$$

and $a_{p \sigma}^{+}$and $a_{q \sigma}$ are the usual spin-orbital creation and annihilation operators. The operator $E_{p q}^{-}$occurs in spin dependent terms which are proportional to the vector-operator component $S_{0}$ of the electron spin. Such operators conserve the total spin.

The MR-CI energy is given by:

$$
E_{\text {MRCI }}=\left\langle\Psi_{\text {MRCI }}\left|\hat{H}^{(0)}\right| \Psi_{\text {MRCI }}\right\rangle=\hat{V}_{N N}+\sum_{p, q} P_{p q}^{+} h_{p q}+\sum_{p, q, r, s} \Gamma_{p q r s}(p q \mid r s)
$$

with the first order reduced electron ('+’) and spin ('-`) density matrices:

$$
P_{p q}^{ \pm}=\left\langle\Psi_{M R C I}\left|E_{p q}^{ \pm}\right| \Psi_{M R C I}\right\rangle=\sum_{I, J} C_{I}^{*} C_{J}\left\langle\Phi_{I}\left|E_{p q}^{ \pm}\right| \Phi_{J}\right\rangle
$$

The spinless second order reduced density matrix is:

$$
\Gamma_{p q r s}=\frac{1}{2}\left\langle\Psi_{M R C I}\left|E_{p q}^{+} E_{r s}^{+}-\delta_{q r} E_{p s}^{+}\right| \Psi_{M R C I}\right\rangle=\frac{1}{2} \sum_{I, J} C_{I}^{*} C_{J}\left\langle\Phi_{I}\left|E_{p q}^{+} E_{r s}^{+}-\delta_{q r} E_{p s}^{+}\right| \Phi_{J}\right\rangle
$$


In the presence of several true or effective one-electron perturbations $\lambda, \mu$,... the first derivative of the MR-CI energy with respect to $\lambda$ becomes:

$$
\left.\frac{\partial E_{M R C I}}{\partial \lambda}\right|_{\lambda=0}=\left\langle\Psi_{M R C I}\left|\frac{\partial \hat{H}}{\partial \lambda}\right| \Psi_{M R C I}\right\rangle
$$

Assuming that the basis functions do not depend on the perturbation and neglecting the orbital relaxation the derivative becomes straightforward:

$$
\left.\frac{\partial E_{M R C I}}{\partial \lambda}\right|_{\lambda=0}=\sum_{p, q} h_{p q}^{(\lambda)} P_{p q}^{ \pm}
$$

The second derivative is then given by:

$$
\left.\frac{\partial^{2} E_{M R C I}}{\partial \lambda \partial \mu}\right|_{\lambda=\mu=0}=\sum_{p, q} h_{p q}^{(\lambda ; \mu)} P_{p q}^{ \pm}+\left.\sum_{p, q} h_{p q}^{(\lambda)} \frac{\partial P_{p q}^{ \pm}}{\partial \mu}\right|_{\mu=0}
$$

where

$$
\begin{aligned}
& h_{p q}^{(\lambda)}=\left.\frac{\partial h_{p q}}{\partial \lambda}\right|_{\lambda=0} \\
& h_{p q}^{(\lambda, \mu)}=\left.\frac{\partial^{2} h_{p q}}{\partial \lambda \partial \mu}\right|_{\lambda=\mu=0}
\end{aligned}
$$

The electron density $\left(\mathbf{P}^{+}\right)$is to be used if the perturbation is spin-independent while a spin-

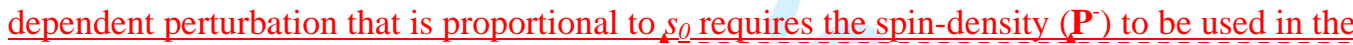
above equations. Based on the arguments of McWeeny in an effective Hamiltonian framework the two-terms in eq (9) may be referred to as 'first' and 'second' order respectively.[30] The first order term is an expectation like quantity that only requires the unperturbed ground state electron or spin density. The second term on the other hand requires $\underline{\text { the calculation of the perturbed electron- or spin density and in a sum-over-states or effective }}$ Hamiltonian picture arises from an infinite sum over excited states, In the case of the MR-CI method, the calculation of the perturbed electron and spin-densities is particularly one requires the response with respect to one of the external perturbations to be evaluated straightforward:

$$
\left.\frac{\partial P_{p q}^{ \pm}}{\partial \mu}\right|_{\mu=0}=\left\langle\frac{\partial \Psi_{M R C I}}{\partial \mu}\left|E_{p q}^{ \pm}\right| \Psi_{M R C I}\right\rangle+\left\langle\Psi_{M R C I}\left|E_{p q}^{ \pm}\right| \frac{\partial \Psi_{M R C I}}{\partial \mu}\right\rangle
$$


The perturbed CI coefficients are obtained from the coupled-perturbed MRCI (CP-MRCI) equations:

$$
\left(\mathbf{H}^{(0)}-\mathbf{1} E_{M R C I}\right) \mathbf{C}^{(\mu)}+\left(\mathbf{H}^{(\mu)}-\mathbf{1}\left\langle\Psi_{M R C I}\left|\hat{H}^{(\mu)}\right| \Psi_{M R C I}\right\rangle\right) \mathbf{C}^{(0)}=0
$$

Here $\mathbf{C}^{(\mu)}$ denotes the perturbed CI coefficients, $\mathbf{H}^{(0)}$ the unperturbed matrix of the BornOppenheimer Hamiltonian in the CSF basis, $E_{M R C I}$ the unperturbed MR-CI energy, $\mathbf{H}^{(\mu)}$ the matrix of the derivative of the total perturbed Hamiltonian with respect to the perturbation parameter evaluated at the origin and $\left\langle\Psi_{M R C I}\left|\hat{H}^{(\mu)}\right| \Psi_{M R C I}\right\rangle$ is the first-order perturbation energy (which is zero for the magnetic field perturbations considered in this work). The set of CP-MRCI equations (eq (13)) constitute a large set of linear equations of a similar structure as the MRCI eigenvalue equation. Consequently, the CP-MRCI equations can be solved by almost identical iterative techniques. The only moderately more complicated feature is the calculation of spin-dependent matrix elements in the calculation of the inhomogeneity which differs substantially from the corresponding terms in the analytic MR-CI gradient.[31] Our implementation of general spin-dependent MRCI matrix elements has been described previously.[32]

If one introduces the spectral resolution of the matrix $\mathbf{H}^{(0)}-1 E_{M R C I}$ one can write:

$$
\left|\frac{\partial \Psi_{M R C I}}{\partial \mu}\right\rangle=-\sum_{K \neq 0} \frac{\left\langle\Psi_{K}\left|\hat{H}^{(\mu)}\right| \Psi_{M R C I}\right\rangle}{E_{K}-E_{M R C I}}\left|\Psi_{K}\right\rangle
$$

where $\left|\Psi_{K}\right\rangle=\sum_{L} C_{L K}^{(0)}\left|\Phi_{L}\right\rangle$ is the $K$ 'th eigenstate of $\hat{H}^{(0)}$ with energy $E_{K}$. This is merely a reminder that the solution of the linear-equation system is equivalent to an untruncated SOS expansion and is thus preferable over truncated SOS approaches.

In the case of the g-tensor the only second-order contribution arises from the terms which are linear in the magnetic field and the electron spin but involve no other spins. These are the orbital-Zeeman operator and the SOC term (e.g. ref [5]). In this work, we take the SOC

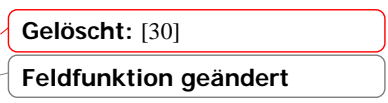

Gelöscht: [31]

Feldfunktion geändert

Formatiert: Schriftart: Kursiv

Feldfunktion geändert 
operator to be an effective one-electron operator and evaluate two approximations to the full Breit-Pauli form of the SOC. The first is the simply effective nuclear charge model as parameterized by Koseki et al.[33-35] It’s matrix elements are given by:

$$
\left\langle p\left|\hat{z}_{\mu}^{(e f f)}\right| q\right\rangle=\frac{\alpha^{2}}{2} \sum_{A} Z_{A}^{(e f f)}\left\langle p\left|r_{A}^{-3} \mathbf{l}_{A \mu}\right| q\right\rangle
$$

with $\alpha \approx 1 / 137$ being the fine-structure constant and $Z_{A}^{(e f f)}$ a semiempirical effective nuclear charge of atom $A$ which were not changed from their original values. $\hat{\mathbf{l}}_{A ; \mu}$ is the $\mu$ 'th component of the angular momentum of the electron relative to atom $\mathrm{A}$ and $r_{\mathrm{A}}$ is the electron's distance to this atom. The second form of the SOC is the sophisticated spin-orbitmean-field (SOMF) approximation introduced by Hess et al.36] (see also the AMFI program [37]) The ORCA implementation of this operator [38] is equivalent to the formulation of Berning et al. [39] and leads to an effective one-electron operator of the form:

$$
\left\langle p\left|\hat{\mathbf{z}}_{\mu}^{(S O M F)}\right| q\right\rangle=\frac{\alpha^{2}}{2} \sum_{A} Z_{A}\left\langle p\left|r_{A}^{-3} \mathbf{l}_{A \mu}\right| q\right\rangle-\frac{\alpha^{2}}{2} \sum_{x, y} P_{x y}\left[\left(p q\left|\hat{g}_{\mu}^{S O C}\right| x y\right)-\frac{3}{2}\left(p x\left|\hat{g}_{\mu}^{S O C}\right| q y\right)-\frac{3}{2}\left(y q\left|\hat{g}_{\mu}^{S O C}\right| p x\right)\right]
$$

with $\hat{g}_{\mu}^{S O C}=r_{12}^{-3} \hat{\mathbf{l}}_{12, \mu}$. Here, $P_{x y}$ is the atomic orbital basis representation of some density (taken here as the MRCI ground state density matrix), $r_{12}$ is the interelectronic distance and $\hat{\mathbf{l}}_{12 ; \mu}$ is the $\mu$ 'th component of the angular momentum of electron 1 relative to electron 2 . In this work, all two-electron integrals were evaluated exactly. The second-order contribution to the g-tensor becomes:

$$
\Delta g_{\mu \nu}^{(O Z / S O C)}=-\frac{1}{2 S} \sum_{p, q} \frac{\partial P_{p q}^{-}}{\partial B_{\mu}}\left\langle p\left|\hat{z}_{v}\right| q\right\rangle
$$

In the theory of the g-tensor, there are three operators which are bilinear in the external magnetic field and the electron spin and therefore contribute to the first-order term in eq (9). These, are the spin-Zeeman term, the reduced mass correction and the gauge-correction term which lead to the following contributions:

$$
g_{\mu v}^{(s Z)}=\delta_{\mu v} g_{e}
$$
$g_{e}=2.002319 \ldots$ is the free electron g-value. The reduced mass correction (RMC) is given by: 


$$
g_{\mu \nu}^{(R M C)}=\delta_{\mu v} \frac{\alpha^{2}}{2 S} \sum_{p, q} P_{p q}^{-}\left\langle p\left|\nabla^{2}\right| q\right\rangle
$$

where $\alpha$ is the fine structure constant and $S$ the total spin of the ground state. In the effective nuclear charge approximation the gauge correction (GC) is given by:

$$
\Delta g_{\mu \nu}^{(G C)}=\frac{\alpha^{2}}{4 S} \sum_{p, q} P_{p q}^{-}\left\langle p\left|\sum_{A} Z_{A}^{(e f f)} r_{A}^{-3}\left\{\mathbf{r}_{A} \mathbf{r}-r_{A, \mu} r_{v}\right\}\right| q\right\rangle
$$

Owing to its smallness of this term and the relatively good performance of the effective nuclear charge model ( $\approx \pm 10 \%$, see below) it did not seem appropriate to implement a more sophisticated approximation.

The method presented above is referred to below as LRT-MRCI treatment.

\section{Computational Details}

All calculations were carried out with the ORCA electronic structure program $[40]$ which has been modified in this work to carry out CP-MRCI calculations. Presently, the program can deal with either purely real or purely imaginary perturbations which may or may not be spindependent. However, the introduction of orbital relaxation is presently not implemented and is also not straightforward since the program makes no assumption about the identity of the input orbitals. For this purpose a more dedicated code seems preferable. Unless otherwise noted, the calculations employed the aug-cc-pVDZ basis set [41], The orbitals were obtained Feldfunktion geändert in tightly converged CASSCF calculations using the reference spaces listed in Table 1. All configurations in the CAS space including those of symmetry different from the ground state were used as reference configurations in the following uncontracted MRCI calculations. All unique single- and double-excitations relative to all reference configurations were included in the variational calculation without selection. Core electrons were not correlated. The linear equation system for the perturbed MRCI coefficients were solved by Pople’s algorithm [42] Feldfunktion geändert Gelöscht: [41] and usually converged in less than 10 iterations to seven digits accuracy. The origin was chosen at the centre of the MRCI electronic charge. 


\section{Results and Discussion}

In general, the MRCI energies in Table 1 are 1-3 mEh higher than the corresponding numbers from spin-unrestricted coupled-cluster calculations with single- and double-excitations together with a perturbative triple-excitations correction $(\operatorname{CCSD}(T))$. If it is assumed that the CCSD(T) results are close to full CI values, the main source of this discrepancy is very likely the size consistency error of the MRCI method. It would be remedied by the multireference Davidson correction. However, since the response of the (nonvariational) Davidson correction [43] has not been coded this would not have affected the outcome of the property calculations. Basis set convergence and core-correlation. Since the basis set convergence of the g-tensor has never been systematically studied at a correlated $a b$ initio level, the g-tensor of the $\mathrm{NH}$ molecule was investigated with the converging series of correlation consistent basis sets. As becomes evident from Table 2, the first order terms are easily converged to about $1 \mathrm{ppm}$ already at the triple- $\zeta$ level. This is, however, not true for the second order PSO term. Here, convergence is as slow as for the dynamic correlation energy itself and even upon going from cc-PVQZ to cc-pV5Z changes on the order of 20 ppm are observed. The data in Table 2 fit reasonably well to a $\mathrm{X}^{-3}$ dependence of the $g_{\perp}^{(P S O)}$ shift with $\mathrm{X}$ being the cardinal number of the basis set. This fit leads to a predicted basis set limit of $\sim 1610 \mathrm{ppm}$ for the $g_{\perp}^{(P S O)}$-value of $\mathrm{NH}$ at the unrelaxed LRT-MRCI level in conjunction with the effective nuclear charge SOC operator. The effects of core-correlation were also briefly investigated for the NH molecule.

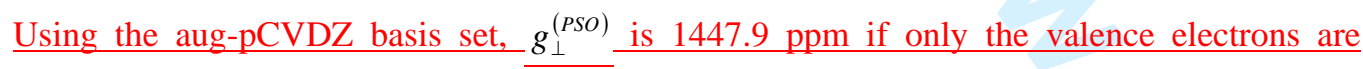
correlated and 1479.5 ppm upon including the $\mathrm{N}-1$ s orbital in the correlation treatment. Thus, at least in this example, the core-correlation effects appear to be limited and will only play an important role upon approaching the basis set limit. The core-correlation effects are also not expected to be large since, unlike the case met in the calculation of the Fermi-contact interaction, the limited core-level spin-polarization is not expected to play an important role 
for the spin-orbit coupling of the valence shell electrons. Nevertheless, this subject certainly warrants a much more careful investigation on a larger test set of molecules including also heavier elements.

Comparison to experiment and other calculations. Comparison of the present calculations to experimental data is of limited value since small basis sets are used here and the experimental data are uncertain and often significantly affected by matrix effects. Hence, comparison can be made to previous SOS calculations of Brownridge et al.,[16] Bolvin [23] and the present author.[18] However, all of the previous calculations used larger basis sets and introduced additional approximations leading to additional uncertainties. Nevertheless, the results of the LRT-MRCI calculations do agree reasonably well with the previous SOS-MRCI calculations except for $\mathrm{H}_{2} \mathrm{O}^{+}$and $\mathrm{AlO}$. The latter case is particularly pathological and has recently been analyzed in much more detail by Gilka and Marian.[44] In this work, the LRT-MRCI calculations for $\mathrm{AlO}$ give reasonable results that appear to be stable.

For $\mathrm{H}_{2} \mathrm{O}^{+}$the results of the LRT-MRCI calculations seem to be inferior to the earlier SOSMRCI calculations (the $\mathrm{g}_{\max }$ shift is predicted too low in the present calculations).[12,16,18,23] The problems appear to be associated with the CASSCF orbitals as will be discussed in more detail below.

Spin orbit operators. It is evident from Table 3 - Table 5 that the effective nuclear charge and SOMF SOC Hamiltonians provide results within $\sim 10 \%$ of each other. The motivation for including the less rigorous effective nuclear charge values is that this operator is easily implemented and this will facilitate future comparison of theoretical g-tensor results. Since the effective nuclear charge values always overshoot the more rigorous SOMF results, it would have been possible to improve on the effective nuclear charge model by dividing the effective charges by 1.1-1.2. However, this would be a merely cosmetic operation.

Discussion of Approximations. In obtaining the theoretical results of this work only two approximations above the limitations of a finite (small) one-particle have been made: (1) 
neglect of the dependence of the basis set on the (magnetic field) perturbation and (2) neglect of the orbital relaxation contribution. The first approximation is not very severe as all calculations to date show that the gauge dependence of the g-tensor is small. Hence, the enormous effort to implement gauge-including atomic orbitals (GIAO's) into the LRT-MRCI code does not seem worthwhile at this stage. Concerning the second approximation, it is obvious that the orbital relaxation contributions must vanish for a full CI wavefunction.[28] To the extent that one hopes to approximate this limit well in the present calculations it could also be hoped that the orbital relaxation contributions are small enough to be neglected. Indeed, in CC calculations of response properties the orbital response is often neglected in order to avoid spurious poles that are introduced via the Hartree-Fock ground state (see for example the interesting discussion in ref [45]). Orbital relaxation contributions are implicitly accounted for in such calculations through the higher powers of the $t_{1}$ (single-excitation cluster) operator. A similar situation does, however, not exist in the case of CI calculations which are linear in the single excitation amplitudes. Thus, if the orbitals are optimized for the ground state, it might happen that the excited states are not well described by the same orbitals and consequently, one might expect that the linear response treatment would predict g-shifts that are too small in magnitude.

In order to find out how large these effects are in the case of g-tensors, calculations with different sets of orbitals were carried out for $\mathrm{NH}, \mathrm{CN}$ and $\mathrm{H}_{2} \mathrm{O}^{+}$. For this purpose it is sufficient to focus on the largest PSO contribution to the g-shift. For NH the largest contribution to the $g_{\perp}^{(P S O)}$-shift in a SOS picture arises from the low-lying ${ }^{3} \Pi$ state. Thus, it seems appropriate to compare calculations where the reference orbitals have been obtained either from single root $\operatorname{CASSCF}(6,5)$ and state-averaged (SA) three-root CASSCF(6,5) calculations. The resulting $g_{\perp}^{(P S O)}$ values are $1418.9 \mathrm{ppm}$ and $1461.3 \mathrm{ppm}$ which amounts to a change of less than $3 \%$. Similarly, for the CN-radical it was shown previously, that the largest
Feldfunktion geändert 
contribution to $g_{\perp}^{(P S O)}$ does not arise from the low-lying $1-^{2} \Pi$ state but from the much higher $2-{ }^{2} \Pi$ state since couples much more strongly to the ground state than $1-{ }^{2} \Pi$.[18] In fact, orbitals from a CASSCF $(9,8)$ yield $g_{\perp}^{(P S O)}=-1889 \mathrm{ppm}$ and a seven root average, which includes the $2-{ }^{2} \Pi$ state yields $g_{\perp}^{(P S O)}=-1882 \mathrm{ppm}$. This result indicates that orbital relaxation effects are also limited in this system.

However, one should not jump to the conclusion that the dependence of the results on the orbitals is always small. In fact, this is not the case - turning to $\mathrm{H}_{2} \mathrm{O}^{+}$, the largest contribution to $g_{33}^{(P S O)}$ arises from the low-lying $1-{ }^{2} \mathrm{~A}_{1}$ state that is experimentally found around 16,500 $\mathrm{cm}^{-1}$.[12] In this system orbital averaging makes a very large difference. Determining the orbitals from a single root CASSCF $(7,6)$ calculation yields a LRT-MRCI value $g_{33}^{(P S O)}=12596$ ppm while two root averaging gives $g_{33}^{(P S O)}=15482 \mathrm{ppm}-$ a change as large as $20 \%$. The seemingly better result of the two-root calculation (the experimental value for the $g_{33}^{(t o t)}$ shift is 18800 ppm [12]) may well be associated with the averaged orbitals that describe the crucial ${ }^{2} \mathrm{~B}_{1} \rightarrow{ }^{2} \mathrm{~A}_{1}$ excitation in a more balanced way than the single root orbitals. In fact, already the SA-CASSCF transition energy of the two-root calculation $\left(18500 \mathrm{~cm}^{-1}\right)$ is close to experiment while the orbitals of the single-root optimization yield $24900 \mathrm{~cm}^{-1}$ which is much too high. This shortcoming is not repaired at the MRCI level, where the transition energy is still predicted too high with the single root orbitals $\left(20400 \mathrm{~cm}^{-1}\right.$ ) while the corresponding value with the two-root orbitals is accurate $\left(16400 \mathrm{~cm}^{-1}\right)$. This shows once more that it is important for linear response treatments of ground state properties that the eigenvalues of the response matrix (for LRT-MRCI simply $\mathbf{H}^{(0)}-\mathbf{1} E_{M R C I}$ ) describe excitation energies well. It is noted in passing that the MRCI calculation based on the two-root CASSCF orbitals even yield a MRCI energy that is slightly lower $(0.2 \mathrm{mEh})$ than the MRCI solution based on the singleroot CASSCF orbitals. Whether a LRT-MRCI calculation that includes orbital relaxation is 
able to compensate for the shortcomings of the single-root CASSCF orbitals appears to be an open question. It is noted, however, that the significant deviations from experiment that still persist are not due to basis set effects. Calculations with the cc-pVQZ basis and the SOMF operator still yield a $g_{33}^{(\text {PSO })}$ of only $\sim 15616$ ppm which is $\sim 20 \%$ below the experimental gasphase value.

\section{Conclusions}

In the present work the first analytic derivative implementation of electronic g-tensors at the MRCI level is reported and has been evaluated through benchmark calculations on small molecules. For a given set of molecular orbitals, the present LRT-MRCI approach is to be preferred over SOS based treatments since the entire manifold of excited states is implicitly accounted for and no truncation error arises. Under the reasonable assumption that the solution of the CP-MRCI equations is computationally about as expensive as the calculation of a single MRCI root, the LRT-MRCI method is also computationally more economical than the SOS-MRCI treatment.

However, the present implementation is not complete in the sense that the orbital relaxation contributions are neglected. While it was initially hoped that the MRCI wavefunction might be accurate enough in order to compensate for this neglect, the actual calculations suggest that this is probably at least not always the case - different sets of state averaged CASSCF orbitals have yielded very different response contributions to the g-tensor for $\mathrm{H}_{2} \mathrm{O}^{+}$. In situations with low lying excited states that strongly spin-orbit couple to the electronic ground state it may prove advantageous to determine the orbitals for an average of several low-lying states since this will allow for a more balanced treatment of excitation energies at the linear response level. For future high-accuracy studies it appears desirable to include the orbital response in such calculations. This requires the solution of the coupled-perturbed CASSCF equations and their derivatives for a purely imaginary perturbation. The development of an efficient 
computer code for this task is a significant undertaking that has so far not been completed in the framework of the ORCA program.

The main fields of application for such an involved methodology might be: (a) accurate calculations on small molecules (owing to the slow basis set convergence this will likely also require basis set extrapolation to be carried out), (b) generation of benchmark values for more approximate schemes and (c) application to molecules with genuine multireference character. The lack of size consistency is a serious issue in such calculations and might be dealt with through average coupled pair functionals.[46] Once these tasks are accomplished, additional approximations must be introduced in order to make LRT-MRCI g-tensor calculations computationally feasible for at least medium sized molecules. Work in this direction is in progress in our group.

Acknowledgement. It is my pleasure to dedicate this paper to Prof. Peter Pulay in recognition of his outstanding contributions to quantum chemistry. I also gratefully acknowledge financial support from the german science foundation (DFG priority program 1137, SFB 624 and SFB Gelöscht: DFG 663), the German-Israeli foundation and the university of Bonn. 
Table 1: Geometries (in a.u. and degrees), CASSCF energies, MRCI energies and CCSD(T) energies (all in Eh)obtained for the test set in the aug-cc-pVDZ basis.

\begin{tabular}{|l|c|c|c|l|l|}
\hline Molecule & E(CASSCF) & E(MR-CI) & E(CCSD(T)) & Ref-Space & Geometry \\
\hline $\mathrm{CN}$ & -92.351608 & -92.501359 & -92.502281 & $\mathrm{CAS}(9,8)$ & $\mathrm{R}_{\mathrm{CN}}=2.2140$ \\
\hline $\mathrm{CO}^{+}$ & -112.423319 & -112.564973 & -112.565419 & $\mathrm{CAS}(9,8)$ & $\mathrm{R}_{\mathrm{CO}}=2.0986$ \\
\hline $\mathrm{BO}$ & -99.651369 & -99.793545 & -99.796694 & $\mathrm{CAS}(9,8)$ & $\mathrm{R}_{\mathrm{BO}}=2.2770$ \\
\hline $\mathrm{AlO}$ & -316.863176 & -317.010044 & -317.011757 & $\mathrm{CAS}(9,8)$ & $\mathrm{R}_{\mathrm{AlO}}=3.0569$ \\
\hline $\mathrm{NH}$ & -54.990463 & -55.103281 & -55.105587 & $\mathrm{CAS}(6,5)$ & $\mathrm{R}_{\mathrm{NH}}=1.9578$ \\
\hline $\mathrm{OH}^{+}$ & -75.005612 & -75.116515 & -75.117897 & $\mathrm{CAS}(6,5)$ & $\mathrm{R}_{\mathrm{OH}}=1.9446$ \\
\hline $\mathrm{PH}$ & -341.301599 & -341.397667 & -341.400433 & $\mathrm{CAS}(6,5)$ & $\mathrm{R}_{\mathrm{PH}}=2.6736$ \\
\hline $\mathrm{SH}^{+}$ & -397.770501 & -397.868444 & -397.870574 & $\mathrm{CAS}(6,5)$ & $\mathrm{R}_{\mathrm{SH}}=2.5965$ \\
\hline $\mathrm{H}_{2} \mathrm{O}^{+}$ & -75.677504 & -75.803890 & -75.806194 & $\mathrm{CAS}(7,6)$ & $\mathrm{R}_{\mathrm{OH}}=2.0893 \angle 109.62$ \\
\hline $\mathrm{NH}_{2}$ & -55.618776 & -55.747935 & -55.751555 & $\mathrm{CAS}(7,6)$ & $\mathrm{R}_{\mathrm{NH}}=1.9291 \angle 103.15$ \\
\hline $\mathrm{H}_{2} \mathrm{~S}^{+}$ & -398.392575 & -398.504049 & -398.506884 & $\mathrm{CAS}(7,6)$ & $\mathrm{R}_{\mathrm{SH}}=2.5614 \angle 93.30$ \\
\hline $\mathrm{PH}_{2}$ & -341.904247 & -342.013942 & -342.017597 & $\mathrm{CAS}(7,6)$ & $\mathrm{R}_{\mathrm{PH}}=2.6663 \angle 91.92$ \\
\hline
\end{tabular}

Table 2: Basis set convergence of LRT-MRCI g-tensor (in ppm) calculations for $\mathrm{NH}\left({ }^{3} \Sigma\right)$ (effective nuclear charge SOC operator).

\begin{tabular}{|l|l|l|l|l|l|l|}
\hline Basis Set & $g_{\|}^{(\text {tot })}$ & $g_{\perp}^{(\text {tot }) \mathrm{a}}$ & $g^{(R M C)}$ & $g_{\|}^{(\text {DSO })}$ & $g_{\perp}^{(D S O)}$ & $g_{\perp}^{(\text {PSO a }}$ \\
\hline cC-pVDZ & -107.4 & 1205.4 & -205.1 & 97.6 & 71.0 & 1339.5 \\
\hline cc-pVTZ & -107.8 & 1394.4 & -205.8 & 98.0 & 70.9 & 1529.2 \\
\hline cc-pVQZ & -107.8 & 1441.6 & -205.8 & 98.0 & 70.9 & 1576.6 \\
\hline cc-pV5Z & -107.8 & 1463.0 & -205.7 & 97.9 & 70.9 & 1597.8 \\
\hline
\end{tabular}

| $\mathrm{a}-\mathrm{h}$ functions had to be deleted due to technical constraints, 
Table 3: MRCI linear response g-tensor (in ppm) results for ${ }^{2} \Sigma$ molecules as described in the text (results obtained with the effective nuclear charge SOC operator. Results obtained with the full SOMF-SOC operator are given in parenthesis).

\begin{tabular}{|l|l|l|l|l|l|l|}
\hline Molecule & $g_{\|}^{(\text {tot })}$ & $g_{\perp}^{(\text {tot }) \mathrm{a}}$ & $g^{(R M C)}$ & $g_{\|}^{(\text {DSO })}$ & $g_{\perp}^{(\text {DSO })}$ & $g_{\perp}^{(\text {PSO a }}$ \\
\hline $\mathrm{CN}$ & -125.7 & $-1946.8(-1758.8)$ & -170.5 & 44.8 & 113.5 & $-1889.8(-1701.8)$ \\
\hline $\mathrm{CO}^{+}$ & -125.4 & $-2361.2(-2144.9)$ & -173.5 & 48.1 & 122.1 & $-2309.8(-2093.6)$ \\
\hline $\mathrm{BO}$ & -61.3 & $-1664.9(-1493.5)$ & -90.8 & 29.5 & 78.8 & $-1652.9(-1481.5)$ \\
\hline $\mathrm{AlO}$ & -78.1 & $-2141.0(-1739.7)$ & -158.7 & 80.6 & 180.4 & $-2162.7(-1761.4)$ \\
\hline
\end{tabular}

Table 4: MRCI linear response g-tensor (in ppm) results for ${ }^{3} \Sigma$ molecules as described in the text (results obtained with the effective nuclear charge SOC operator. Results obtained with the full SOMF-SOC operator are given in parenthesis).

\begin{tabular}{|l|l|l|l|l|l|l|}
\hline Molecule & $g_{\|}^{(\text {tot })}$ & $g_{\perp}^{(\text {tot }) \mathrm{a}}$ & $g^{(\text {RMC })}$ & $g_{\|}^{(\text {DSO })}$ & $g_{\perp}^{(\text {DSO })}$ & $g_{\perp}^{(\text {PSO a }}$ \\
\hline $\mathrm{NH}$ & -106.0 & $1286.6(1131.0)$ & -203.0 & 97.0 & 70.6 & $1418.9(1263.4)$ \\
\hline $\mathrm{OH}^{+}$ & -175.5 & $3619.9(3260.9)$ & -323.7 & 148.2 & 109.8 & $3822.8(3474.8)$ \\
\hline $\mathrm{PH}$ & -17.8 & $4201.5(3823.2)$ & -146.0 & 163.7 & 117.1 & $4230.4(3852.1)$ \\
\hline $\mathrm{SH}^{+}$ & -19.1 & $8068.6(7609.6)$ & -229.8 & 210.8 & 152.6 & $8145.9(7686.9)$ \\
\hline
\end{tabular}


Table 5: MRCI linear response g-tensor (in ppm) results for ${ }^{2} \mathrm{XH}_{2}{ }^{\mathrm{n}}(\mathbf{n}=\mathbf{0 , 1})$ molecules with ${ }^{2} \mathrm{~B}_{1}$ ground states as described in the text (results obtained with the effective nuclear charge SOC operator. Results obtained with the full SOMF-SOC operator are given below).

\begin{tabular}{|c|c|c|c|c|c|c|c|c|c|c|}
\hline Molecule & $g_{11}^{(t o t)}$ & $g_{22}^{(t o t)}$ & $g_{33}^{(t o t)}$ & $g^{(R M C)}$ & $g_{11}^{(\mathrm{DSO})}$ & $g_{22}^{(\mathrm{DSO})}$ & $g_{33}^{(\mathrm{DSO})}$ & $g_{11}^{(P S O)}$ & $g_{22}^{(P S O)}$ & $g_{33}^{(P S O)}$ \\
\hline \multirow[t]{2}{*}{$\mathrm{H}_{2} \mathrm{O}^{+}$} & -191.2 & 4566.3 & 12427.1 & -317.5 & 73.2 & 146.7 & $\begin{array}{l}147.9 \\
\end{array}$ & 53.1 & 4737.1 & 12598.3 \\
\hline & -198.1 & 4006.9 & 11117.0 & & & & & 46.3 & 4177.7 & 11286.7 \\
\hline \multirow[t]{2}{*}{$\mathrm{NH}_{2}$} & -144.9 & 1532.3 & 4767.1 & -198.3 & 45.9 & 97.1 & 98.6 & 7.5 & 1633.5 & 4866.9 \\
\hline & -147.9 & 1312.7 & 4183.9 & & & & & 4.6 & 1414.0 & 4283.6 \\
\hline \multirow{2}{*}{$\mathrm{H}_{2} \mathrm{~S}^{+}$} & 41.3 & 10698.9 & 24088.9 & -228.7 & 97.6 & 212.0 & 210.3 & 172.4 & 10715.6 & 24107.3 \\
\hline & 24.0 & 10071.1 & 22727.0 & & & & & 155.1 & 10087.8 & 22745.4 \\
\hline \multirow[t]{2}{*}{$\mathrm{PH}_{2}$} & 24.6 & 4707.4 & 13622.3 & -146.1 & 74.1 & 165.6 & 164.0 & 96.6 & 4687.9 & 13604.5 \\
\hline & 8.9 & 4272.5 & 12409.6 & & 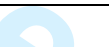 & & & 80.9 & 4252.9 & 12391.8 \\
\hline
\end{tabular}




\section{Literature}

[1] F. Neese, in: B. Gilbert (Ed.), Specialist Reports on EPR Spectroscopy. Royal Society of Chemistry, London, 2007, p. 73.

[2] F. Neese Biol. Mag. Res. (Ed. G. Hanson) (2007) in press.

[3] S. Patchkovskii, G. Schreckenbach, in: M. Kaupp, V. Malkin, M. Bühl (Eds.), Calculation of NMR and EPR Parameters. Wiley-VCH, Weinheim, 2004, p. 505.

[4] G. Schreckenbach, T. Ziegler J. Phys. Chem. A. 101 (1997) 3388.

[5] F. Neese J. Chem. Phys. 115 (2001) 11080.

[6] D. Jayatilaka J. Chem. Phys 108 (1998) 7587.

[7] P. Bündgen, G. H. Lushington, F. Grein Int. J. Quant. Chem. 29 (1995) 283.

[8] G. H. Lushington, P. Bündgen, F. Grein Int. J. Quant. Chem. 55 (1995) 377.

[9] P. J. Bruna, G. H. Lushington, F. Grein Chem. Phys. Lett. 258 (1996) 427.

[10] G. H. Lushington, F. Grein Theor. Chim. Acta 93 (1996) 259.

[11] G. H. Lushington, F. Grein Int. J. Quant. Chem.: Quant. Chem. Symp. 30 (1996) 467.

[12] G. H. Lushington, F. Grein J. Chem. Phys. 106 (1997) 3292.

[13] P. J. Bruna, F. Grein J. Chem. Phys. 109 (1998) 9439.

[14] P. J. Bruna, F. Grein Int. J. Quant. Chem 77 (2000) 324.

[15] P. J. Bruna, F. Grein Chem. Phys. Lett. 318 (2000) 263.

[16] S. Brownridge, F. Grein, J. Tatchen, M. Kleinschmidt, C. M. Marian J. Chem. Phys. 118 (2003) 9552.

[17] F. Neese Mag. Res. Chem. 42 (2004) S187.

[18] F. Neese Chem. Phys. Lett. 380 (2003) 721.

[19] F. Neese Int. J. Quant. Chem. 83 (2001) 104.

[20] A. Delabie, K. Pierloot, M. H. Groothaert, B. M. Weckhuysen, R. A. Schoonheydt Phys. Chem. Chem. Phys. 4 (2002) 134.

[21] K. Pierloot, A. Delabie, M. H. Groothaert, R. A. Schoonheydt Phys. Chem. Chem. Phys. 3 (2001) 2174.

[22] H. Bolvin Inorg. Chem. 46 (2007) 417

[23] H. Bolvin Chem. Phys. Chem. 7 (2006) 1575.

[24] O. Vahtras, M. Engström, B. Schimmelpfennig Chem. Phys. Lett. 351 (2002) 424.

[25] M. Engström, O. Vahtras, H. Ågren Chem. Phys. Lett. 243 (1999) 263.

[26] M. Engström, B. Minaev, O. Vahtras, H. Agren Chem. Phys. Lett. 237 (1998) 149.

[27] O. Vahtras, B. Minaev, H. Agren Chem. Phys. Lett. 281 (1997) 186.

[28] M. Kallay, J. Gauss J. Chem. Phys. 120 (2004) 6841.

[29] M. Kallay, J. Gauss, P. G. Szalay J. Chem. Phys. 119 (2003) 2991.

[30] R. McWeeny: Methods of Molecular Quantum Mechanics, Academic press, London, 1992.

[31] H. Lischka, R. Shepard, R. M. Pitzer, I. Shavitt, M. Dallos, T. Muller, P. G. Szalay, M. Seth, G. S. Kedziora, S. Yabushita, Z. Y. Zhang Phys. Chem. Chem. Phys. 3 (2001) 664.

[32] D. Ganyushin, F. Neese J. Chem. Phys. 125 (2006) 024103.

[33] S. Koseki, M. W. Schmidt, M. S. Gordon J. Chem. Phys. 96 (1992) 10768.

[34] S. Koseki, M. S. Gordon, M. W. Schmidt, N. Matsunaga J. Phys. Chem. 99 (1995) 12764

[35] S. Koseki, M. W. Schmidt, M. S. Gordon J. Phys. Chem. A. 102 (1998) 10430.

[36] B. A. Hess, C. M. Marian, U. Wahlgren, O. Gropen Chem. Phys. Lett. 251 (1996) 365.

[37] B. Schimmelpfennig AMFI - an atomic mean field integral program. University of Stockholm, Stockholm, Sweden, 1996.

[38] F. Neese J. Chem. Phys. 122 (2005) 034107/1.
Formatiert: Englisch

(Großbritannien)

Formatiert: Niederländisch

(Niederlande)

Formatiert: Französisch (Frankreich)

Formatiert: Englisch (Großbritannien)

Formatiert: Französisch

(Frankreich)

Formatiert: Niederländisch

(Niederlande)

Formatiert: Französisch

(Frankreich)

Formatiert: Englisch

(Großbritannien)

Formatiert: Schwedisch

(Schweden)

Formatiert: Englisch

(Großbritannien)

Formatiert: Englisch

(Großbritannien) 
[39] A. Berning, M. Schweizer, H. J. Werner, P. J. Knowles, P. Palmieri Molec. Phys. 98 (2000) 1823.

[40] F. Neese ORCA - an ab initio, density functional and semiempirical program package, University of Bonn, Germany, 2007.

[41] J. T. H. Dunning J. Chem. Phys. 90 (1989) 1007.

[42] J. A. Pople, R. Krishnan, H. B. Schlegel, J. S. Binkley Int. J. Quant. Chem.: Quant. Chem. Symp. 13 (1979) 225.

[43] G. Hirsch, P. J. Bruna, S. D. Peyerimhoff, R. J. Buenker Chem. Phys. Lett. 52 (1977) $\underline{442 .}$

[44] N. Gilka, C. M. Marian Chem. Phys. (2007) submitted for publication.

[45] P. G. Szalay, J. Vazquez, C. Simmons, J. F. Stanton J. Chem. Phys. 121 (2004) 7624.

[46] R. J. Gdanitz, R. Ahlrichs Chem. Phys. Lett. 143 (1988) 413.
Formatiert: Englisch

(Großbritannien)

Formatiert: Einzug: Links: 0 $\mathrm{cm}$, Erste Zeile: $0 \mathrm{~cm}$

Gelöscht: [1] F. Neese, in: B. Gilbert (Ed.), Specialist Reports on EPR Spectroscopy. Royal Society of Chemistry, London, 2007, p. 73.ף

[2] . F. Neese Biol. Mag. Res. (Ed.

G. Hanson) (2007) in press.ๆ

[3] S. Patchkovskii, G.

Schreckenbach, in: M. Kaupp, V.

Malkin, M. Bühl (Eds.), Calculation of NMR and EPR Parameters.

Wiley-VCH, Weinheim, 2004, p.

505.ๆ

[4] G. Schreckenbach, T. Ziegler J. Phys. Chem. A. 101 (1997) 3388. [5] . F. Neese J. Chem. Phys. 115 (2001) 11080.ף

[6] . D. Jayatilaka J. Chem. Phys 108 (1998) 7587.9

[7] . P. Bündgen, G. H. Lushington, F. Grein Int. J. Quant. Chem. 29 (1995) 283.ף

[8] G. H. Lushington, P. Bündgen, F. Grein Int. J. Quant. Chem. 55 (1995) 377.

[9] . P. J. Bruna, G. H. Lushington, F. Grein Chem. Phys. Lett. 258 (1996) 427.

[10] G. H. Lushington, F. Grein Theor. Chim. Acta 93 (1996) 259. [11] . G. H. Lushington, F. Grein Int. J. Quant. Chem.: Quant. Chem. Symp. 30 (1996) 467.』

[12] . G. H. Lushington, F. Grein J. Chem. Phys. 106 (1997) 3292.9 [13] . P. J. Bruna, F. Grein J. Chem. Phys. 109 (1998) 9439.

[14] P. J. Bruna, F. Grein Int. J. Quant. Chem 77 (2000) 324.9

[15] . P. J. Bruna, F. Grein Chem. Phys. Lett. 318 (2000) 263.ף

[16] S. Brownridge, F. Grein, J. Tatchen, M. Kleinschmidt, C. M. Marian J. Chem. Phys. 118 (2003) 9552. ๆ

[17] . F. Neese Mag. Res. Chem. 42 (2004) S187.9

[18] F. Neese Chem. Phys. Lett. 380 (2003) 721.9

[19] . F. Neese Int. J. Quant. Chem. 83 (2001) 104.9

[20] A. Delabie, K. Pierloot, M. H. Groothaert, B. M. Weckhuysen, R. A. Schoonheydt Phys. Chem. Chem. Phys. 4 (2002) 134.ף [21] . K. Pierloot, A. Delabie, M. H. Groothaert, R. A. Schoonheydt Phys. Chem. Chem. Phys. 3 (2001) 2174. 9

[22] . H. Bolvin Inorg. Chem. 46 (2007) 417

[23] . H. Bolvin Chem. Phys. Chem. 7 (2006) 1575. .

[24] . O. Vahtras, M. Engström, B. Schimmelpfennig Chem. Phys. Lett. 351 (2002) 424.

[25] . M. Engström, O. Vahtras, H. Ågren Chem. Phys. Lett. 243 (1999) 263.ศ

[26] M. Engström, B. Minaev, O. Vahtras, H. Agren Chem. Phys. Lett. 237 (1998) 149.1

[27] . O. Vahtras, B. Minaev ... [1] 
[1] F. Neese, in: B. Gilbert (Ed.), Specialist Reports on EPR Spectroscopy. Royal Society of Chemistry, London, 2007, p. 73.

[2] F. Neese Biol. Mag. Res. (Ed. G. Hanson) (2007) in press.

[3] S. Patchkovskii, G. Schreckenbach, in: M. Kaupp, V. Malkin, M. Bühl (Eds.), Calculation of NMR and EPR Parameters. Wiley-VCH, Weinheim, 2004, p. 505.

[4] G. Schreckenbach, T. Ziegler J. Phys. Chem. A. 101 (1997) 3388.

[5] F. Neese J. Chem. Phys. 115 (2001) 11080.

[6] D. Jayatilaka J. Chem. Phys 108 (1998) 7587.

[7] P. Bündgen, G. H. Lushington, F. Grein Int. J. Quant. Chem. 29 (1995) 283.

[8] G. H. Lushington, P. Bündgen, F. Grein Int. J. Quant. Chem. 55 (1995) 377.

[9] P. J. Bruna, G. H. Lushington, F. Grein Chem. Phys. Lett. 258 (1996) 427.

[10] G. H. Lushington, F. Grein Theor. Chim. Acta 93 (1996) 259.

[11] G. H. Lushington, F. Grein Int. J. Quant. Chem.: Quant. Chem. Symp. 30 (1996) 467.

[12] G. H. Lushington, F. Grein J. Chem. Phys. 106 (1997) 3292.

[13] P. J. Bruna, F. Grein J. Chem. Phys. 109 (1998) 9439.

[14] P. J. Bruna, F. Grein Int. J. Quant. Chem 77 (2000) 324.

[15] P. J. Bruna, F. Grein Chem. Phys. Lett. 318 (2000) 263.

[16] S. Brownridge, F. Grein, J. Tatchen, M. Kleinschmidt, C. M. Marian J. Chem. Phys. 118 (2003) 9552.

[17] F. Neese Mag. Res. Chem. 42 (2004) S187.

[18] F. Neese Chem. Phys. Lett. 380 (2003) 721.

[19] F. Neese Int. J. Quant. Chem. 83 (2001) 104.

[20] A. Delabie, K. Pierloot, M. H. Groothaert, B. M. Weckhuysen, R. A. Schoonheydt Phys. Chem. Chem. Phys. 4 (2002) 134.

[21] K. Pierloot, A. Delabie, M. H. Groothaert, R. A. Schoonheydt Phys. Chem. Chem. Phys. 3 (2001) 2174.

[22] H. Bolvin Inorg. Chem. 46 (2007) 417

[23] H. Bolvin Chem. Phys. Chem. 7 (2006) 1575.

[24] O. Vahtras, M. Engström, B. Schimmelpfennig Chem. Phys. Lett. 351 (2002) 424.

[25] M. Engström, O. Vahtras, H. Ågren Chem. Phys. Lett. 243 (1999) 263.

[26] M. Engström, B. Minaev, O. Vahtras, H. Agren Chem. Phys. Lett. 237 (1998) 149.

[27] O. Vahtras, B. Minaev, H. Agren Chem. Phys. Lett. 281 (1997) 186.

[28] M. Kallay, J. Gauss J. Chem. Phys. 120 (2004) 6841.

[29] M. Kallay, J. Gauss, P. G. Szalay J. Chem. Phys. 119 (2003) 2991.

[30] H. Lischka, R. Shepard, R. M. Pitzer, I. Shavitt, M. Dallos, T. Müller, P. G. Szalay, M. Seth, G. S. Kedziora, S. Yabushita, Z. Y. Zhang Phys. Chem. Chem. Phys. 3 (2001) 664.

[31] D. Ganyushin, F. Neese J. Chem. Phys. 125 (2006) 024103.

[32] S. Koseki, M. W. Schmidt, M. S. Gordon J. Chem. Phys. 96 (1992) 10768.

[33] S. Koseki, M. S. Gordon, M. W. Schmidt, N. Matsunaga J. Phys. Chem. 99 (1995) 12764.

[34] S. Koseki, M. W. Schmidt, M. S. Gordon J. Phys. Chem. A. 102 (1998) 10430.

[35] B. A. Hess, C. M. Marian, U. Wahlgren, O. Gropen Chem. Phys. Lett. 251 (1996) 365.

[36] B. Schimmelpfennig AMFI - an atomic mean field integral program. University of Stockholm, Stockholm, Sweden, 1996.

[37] F. Neese J. Chem. Phys. 122 (2005) 034107/1.

[38] A. Berning, M. Schweizer, H. J. Werner, P. J. Knowles, P. Palmieri Molec. Phys. 98 (2000) 1823.

[39] F. Neese ORCA - an ab initio, density functional and semiempirical program package, , University of Bonn, Germany, 2007. 
[40] J. T. H. Dunning J. Chem. Phys. 90 (1989) 1007.

[41] J. A. Pople, R. Krishnan, H. B. Schlegel, J. S. Binkley Int. J. Quant. Chem.: Quant. Chem. Symp. 13 (1979) 225.

[42] G. Hirsch, P. J. Bruna, S. D. Peyerimhoff, R. J. Buenker Chem. Phys. Lett. 52 (1977) 442.

[43] N. Gilka, C. M. Marian Chem. Phys. (2007) submitted for publication.

[44] P. G. Szalay, J. Vazquez, C. Simmons, J. F. Stanton J. Chem. Phys. 121 (2004) 7624.

[45] R. J. Gdanitz, R. Ahlrichs Chem. Phys. Lett. 143 (1988) 413. 


\section{Analytic Derivative Calculation of Electronic g-Tensors based on Multireference Configuration Interaction Wavefunctions}

Frank Neese

Lehrstuhl für Theoretische Chemie Institut für Physikalische und Theoretische Chemie

Universität Bonn

Wegelerstr. 12

D-53115 Bonn

Germany

neese@thch.uni-bonn.de

Formatted: English U.K. 
1

2

3

\begin{abstract}
A computer program has been written to perform analytic derivative calculations of magnetic response properties based on uncontracted multireference configuration interaction (MRCI) wavefunctions. At this point orbital relaxation is neglected in this treatment and the basis functions are assumed to be independent of the perturbation. It is therefore equivalent to an untruncated sum-over-states (SOS) approach to the g-tensor. Hence, the treatment is more rigorous than all previous MRCI g-tensor calculations that have used truncated SOS formulations. In the present calculations, MR-CI calculations were performed on top of full valence complete-active space self-consistent field (CASSCF) reference wavefunctions without truncation of the reference or correlation spaces. Results are reported for two types of spin-orbit coupling operators, a simple effective nuclear charge approximation and the spinorbit mean field (SOMF) approximation. The effects of orbital relaxation are discussed. The values obtained can be used in future calibration studies.
\end{abstract}




\section{Introduction}

The theory of the g-tensor, the central property measured in electron paramagnetic resonance (EPR) experiments, has undergone a rapid development in recent years. In particular, methods based on density functional theory (DFT) have become very popular and are now widely applied in the interpretation of experiments (for reviews see [1-3]). However, one difficulty has been to find highly accurate data for calibration purposes since the g-values measured for small molecules in matrix isolation or the gas phase show a large scatter among different investigations (see for example the data assembled in ref [4]). Thus, there is a need for highly accurate reference data to gauge the quality of approximate approaches. In the ab initio field, g-tensors have been previously calculated by analytic derivative UHF theory,[5] generalized Hartree-Fock theory[6] and sum-over-states (SOS) based formulations for restricted-openshell Hartree-Fock (ROHF), multireference configuration-interaction (MRCI) [7-19] as well as multireference perturbation $[18,20-23]$ approaches. In addition, a linear response theory (LRT) for g-tensors based on the complete active space self-consistent field (CASSCF) method has been developed and applied.[24-27]

In this paper, an analytic derivative (linear response) method for the calculation of electronic g-tensors based on MRCI wavefunctions is reported. The method is approximate in thus far, as that orbital relaxation contributions and the dependence of the basis set on the perturbation are neglected. The present development is considered to be important since MRCI treatments are the most general and robust approach to electronic structure theory as they treat both static and dynamic correlation contributions in a balanced way.

\section{Theory}

The theory of analytic first- and second-derivatives for MR-CI wavefunctions is well known and will only be briefly described in order to document what was actually programmed. We basically follow Kallay and Gauss who have recently described general methods to obtain 
analytic first- and second-derivatives for arbitrary CI and coupled-cluster (CC) wavefunctions.[28,29] The former case is considerably simpler since the MRCI energy is stationary with respect to variations in the CI coefficients. The MRCI wavefunction (assumed to be normalized) is given by:

$$
\Psi_{M R C I}=\sum_{I} C_{I}^{(0)} \Phi_{I}
$$

The variational CI coefficients $C_{I}^{(0)}$ (the superscript (0) denotes the unperturbed situation) have been obtained by diagonalizing the Born-Oppenheimer Hamiltonian:

$$
\hat{H}^{(0)}=\hat{V}_{N N}+\sum_{p, q} h_{p q} E_{p q}^{+}+\frac{1}{2} \sum_{p, q, r, s}(p q \mid r s)\left[E_{p q}^{+} E_{r s}^{+}-\delta_{q r} E_{p s}^{+}\right]
$$

in the space of some configuration-state functions (CSFs) $\{\Phi\}$ of the desired spin- and spacesymmetry (e.g. by solving $\left.\left(\mathbf{H}^{(0)}-\mathbf{1} E_{M R C I}\right) \mathbf{C}^{(0)}=0\right) . \hat{V}_{N N}$ is the nuclear repulsion energy, $h_{p q}$ the matrix elements of the one-electron operator and $(p q \mid r s)$ are the two-electron repulsion integrals in charge cloud notation. The orbital replacement operators are defined by:

$$
E_{p q}^{ \pm}=a_{p \alpha}^{+} a_{q \alpha} \pm a_{p \beta}^{+} a_{q \beta}
$$

and $a_{p \sigma}^{+}$and $a_{q \sigma}$ are the usual spin-orbital creation and annihilation operators. The operator $E_{p q}^{-}$occurs in spin dependent terms which are proportional to the vector-operator component $s_{0}$ of the electron spin. Such operators conserve the total spin.

The MR-CI energy is given by:

$$
E_{M R C I}=\left\langle\Psi_{M R C I}\left|\hat{H}^{(0)}\right| \Psi_{M R C I}\right\rangle=\hat{V}_{N N}+\sum_{p, q} P_{p q}^{+} h_{p q}+\sum_{p, q, r, s} \Gamma_{p q r s}(p q \mid r s)
$$

with the first order reduced electron ('+') and spin ('-') density matrices:

$$
P_{p q}^{ \pm}=\left\langle\Psi_{M R C I}\left|E_{p q}^{ \pm}\right| \Psi_{M R C I}\right\rangle=\sum_{I, J} C_{I}^{*} C_{J}\left\langle\Phi_{I}\left|E_{p q}^{ \pm}\right| \Phi_{J}\right\rangle
$$

The spinless second order reduced density matrix is:

$$
\Gamma_{p q r s}=\frac{1}{2}\left\langle\Psi_{M R C I}\left|E_{p q}^{+} E_{r s}^{+}-\delta_{q r} E_{p s}^{+}\right| \Psi_{M R C I}\right\rangle=\frac{1}{2} \sum_{I, J} C_{I}^{*} C_{J}\left\langle\Phi_{I}\left|E_{p q}^{+} E_{r s}^{+}-\delta_{q r} E_{p s}^{+}\right| \Phi_{J}\right\rangle
$$


In the presence of several true or effective one-electron perturbations $\lambda, \mu, \ldots$ the first derivative of the MR-CI energy with respect to $\lambda$ becomes:

$$
\left.\frac{\partial E_{M R C I}}{\partial \lambda}\right|_{\lambda=0}=\left\langle\Psi_{M R C I}\left|\frac{\partial \hat{H}}{\partial \lambda}\right| \Psi_{M R C I}\right\rangle
$$

Assuming that the basis functions do not depend on the perturbation and neglecting the orbital relaxation the derivative becomes straightforward:

$$
\left.\frac{\partial E_{M R C I}}{\partial \lambda}\right|_{\lambda=0}=\sum_{p, q} h_{p q}^{(\lambda)} P_{p q}^{ \pm}
$$

The second derivative is then given by:

$$
\left.\frac{\partial^{2} E_{M R C I}}{\partial \lambda \partial \mu}\right|_{\lambda=\mu=0}=\sum_{p, q} h_{p q}^{(\lambda ; \mu)} P_{p q}^{ \pm}+\left.\sum_{p, q} h_{p q}^{(\lambda)} \frac{\partial P_{p q}^{ \pm}}{\partial \mu}\right|_{\mu=0}
$$

where

$$
\begin{aligned}
& h_{p q}^{(\lambda)}=\left.\frac{\partial h_{p q}}{\partial \lambda}\right|_{\lambda=0} \\
& h_{p q}^{(\lambda, \mu)}=\left.\frac{\partial^{2} h_{p q}}{\partial \lambda \partial \mu}\right|_{\lambda=\mu=0}
\end{aligned}
$$

The electron density $\left(\mathbf{P}^{+}\right)$is to be used if the perturbation is spin-independent while a spindependent perturbation that is proportional to $s_{0}$ requires the spin-density $\left(\mathbf{P}^{-}\right)$to be used in the above equations. Based on the arguments of McWeeny in an effective Hamiltonian framework the two-terms in eq (9) may be referred to as 'first' and 'second' order respectively.[30] The first order term is an expectation like quantity that only requires the unperturbed ground state electron or spin density. The second term on the other hand requires the calculation of the perturbed electron- or spin density and in a sum-over-states or effective Hamiltonian picture arises from an infinite sum over excited states. In the case of the MR-CI method, the calculation of the perturbed electron and spin-densities is particularly straightforward:

$$
\left.\frac{\partial P_{p q}^{ \pm}}{\partial \mu}\right|_{\mu=0}=\left\langle\frac{\partial \Psi_{M R C I}}{\partial \mu}\left|E_{p q}^{ \pm}\right| \Psi_{M R C I}\right\rangle+\left\langle\Psi_{M R C I}\left|E_{p q}^{ \pm}\right| \frac{\partial \Psi_{M R C I}}{\partial \mu}\right\rangle
$$


The perturbed CI coefficients are obtained from the coupled-perturbed MRCI (CP-MRCI) equations:

$$
\left(\mathbf{H}^{(0)}-\mathbf{1} E_{M R C I}\right) \mathbf{C}^{(\mu)}+\left(\mathbf{H}^{(\mu)}-\mathbf{1}\left\langle\Psi_{M R C I}\left|\hat{H}^{(\mu)}\right| \Psi_{M R C I}\right\rangle\right) \mathbf{C}^{(0)}=0
$$

Here $\mathbf{C}^{(\mu)}$ denotes the perturbed CI coefficients, $\mathbf{H}^{(0)}$ the unperturbed matrix of the BornOppenheimer Hamiltonian in the CSF basis, $E_{M R C I}$ the unperturbed MR-CI energy, $\mathbf{H}^{(\mu)}$ the matrix of the derivative of the total perturbed Hamiltonian with respect to the perturbation parameter evaluated at the origin and $\left\langle\Psi_{M R C I}\left|\hat{H}^{(\mu)}\right| \Psi_{M R C I}\right\rangle$ is the first-order perturbation energy (which is zero for the magnetic field perturbations considered in this work). The set of CP-MRCI equations (eq (13)) constitute a large set of linear equations of a similar structure as the MRCI eigenvalue equation. Consequently, the CP-MRCI equations can be solved by almost identical iterative techniques. The only moderately more complicated feature is the calculation of spin-dependent matrix elements in the calculation of the inhomogeneity which differs substantially from the corresponding terms in the analytic MR-CI gradient.[31] Our implementation of general spin-dependent MRCI matrix elements has been described previously.[32]

If one introduces the spectral resolution of the matrix $\mathbf{H}^{(0)}-\mathbf{1} E_{M R C I}$ one can write:

$$
\left|\frac{\partial \Psi_{M R C I}}{\partial \mu}\right\rangle=-\sum_{K \neq 0} \frac{\left\langle\Psi_{K}\left|\hat{H}^{(\mu)}\right| \Psi_{M R C I}\right\rangle}{E_{K}-E_{M R C I}}\left|\Psi_{K}\right\rangle
$$

where $\left|\Psi_{K}\right\rangle=\sum_{L} C_{L K}^{(0)}\left|\Phi_{L}\right\rangle$ is the $K^{\prime}$ th eigenstate of $\hat{H}^{(0)}$ with energy $E_{K}$. This is merely a reminder that the solution of the linear-equation system is equivalent to an untruncated SOS expansion and is thus preferable over truncated SOS approaches.

In the case of the g-tensor the only second-order contribution arises from the terms which are linear in the magnetic field and the electron spin but involve no other spins. These are the orbital-Zeeman operator and the SOC term (e.g. ref [5]). In this work, we take the SOC 
operator to be an effective one-electron operator and evaluate two approximations to the full Breit-Pauli form of the SOC. The first is the simply effective nuclear charge model as parameterized by Koseki et al.[33-35] It's matrix elements are given by:

$$
\left\langle p\left|\hat{z}_{\mu}^{(e f f)}\right| q\right\rangle=\frac{\alpha^{2}}{2} \sum_{A} Z_{A}^{(e f f)}\left\langle p\left|r_{A}^{-3} \mathbf{l}_{A \mu}\right| q\right\rangle
$$

with $\alpha \approx 1 / 137$ being the fine-structure constant and $Z_{A}^{(e f f)}$ a semiempirical effective nuclear charge of atom $A$ which were not changed from their original values. $\hat{\mathbf{l}}_{A ; \mu}$ is the $\mu^{\prime}$ th component of the angular momentum of the electron relative to atom $\mathrm{A}$ and $r_{A}$ is the electron's distance to this atom. The second form of the SOC is the sophisticated spin-orbitmean-field (SOMF) approximation introduced by Hess et al.[36] (see also the AMFI program [37]) The ORCA implementation of this operator [38] is equivalent to the formulation of Berning et al. [39] and leads to an effective one-electron operator of the form:

$$
\left\langle p\left|\hat{z}_{\mu}^{(\text {SOMF })}\right| q\right\rangle=\frac{\alpha^{2}}{2} \sum_{A} Z_{A}\left\langle p\left|r_{A}^{-3} \mathbf{l}_{A \mu}\right| q\right\rangle-\frac{\alpha^{2}}{2} \sum_{x, y} P_{x y}\left[\left(p q\left|\hat{g}_{\mu}^{S O C}\right| x y\right)-\frac{3}{2}\left(p x\left|\hat{g}_{\mu}^{S O C}\right| q y\right)-\frac{3}{2}\left(y q\left|\hat{g}_{\mu}^{S O C}\right| p x\right)\right]
$$

with $\hat{g}_{\mu}^{S O C}=r_{12}^{-3} \hat{\mathbf{l}}_{12, \mu}$. Here, $P_{x y}$ is the atomic orbital basis representation of some density (taken here as the MRCI ground state density matrix), $r_{12}$ is the interelectronic distance and $\hat{\mathbf{I}}_{12 ; \mu}$ is the $\mu^{\prime}$ th component of the angular momentum of electron 1 relative to electron 2 . In this work, all two-electron integrals were evaluated exactly. The second-order contribution to the g-tensor becomes:

$$
\Delta g_{\mu \nu}^{(O Z / S O C)}=-\frac{1}{2 S} \sum_{p, q} \frac{\partial P_{p q}^{-}}{\partial B_{\mu}}\left\langle p\left|\hat{z}_{v}\right| q\right\rangle
$$

In the theory of the g-tensor, there are three operators which are bilinear in the external magnetic field and the electron spin and therefore contribute to the first-order term in eq (9). These, are the spin-Zeeman term, the reduced mass correction and the gauge-correction term which lead to the following contributions:

$$
g_{\mu \nu}^{(s Z)}=\delta_{\mu \nu} g_{e}
$$

$g_{e}=2.002319 \ldots$ is the free electron g-value. The reduced mass correction (RMC) is given by: 


$$
g_{\mu \nu}^{(R M C)}=\delta_{\mu \nu} \frac{\alpha^{2}}{2 S} \sum_{p, q} P_{p q}^{-}\left\langle p\left|\nabla^{2}\right| q\right\rangle
$$

where $\alpha$ is the fine structure constant and $S$ the total spin of the ground state. In the effective nuclear charge approximation the gauge correction (GC) is given by:

$$
\Delta g_{\mu \nu}^{(G C)}=\frac{\alpha^{2}}{4 S} \sum_{p, q} P_{p q}^{-}\left\langle p\left|\sum_{A} Z_{A}^{(e f f)} r_{A}^{-3}\left\{\mathbf{r}_{A} \mathbf{r}-r_{A, \mu} r_{\nu}\right\}\right| q\right\rangle
$$

Owing to its smallness of this term and the relatively good performance of the effective nuclear charge model $(\approx \pm 10 \%$, see below) it did not seem appropriate to implement a more sophisticated approximation.

The method presented above is referred to below as LRT-MRCI treatment.

\section{Computational Details}

All calculations were carried out with the ORCA electronic structure program [40] which has been modified in this work to carry out CP-MRCI calculations. Presently, the program can deal with either purely real or purely imaginary perturbations which may or may not be spindependent. However, the introduction of orbital relaxation is presently not implemented and is also not straightforward since the program makes no assumption about the identity of the input orbitals. For this purpose a more dedicated code seems preferable. Unless otherwise noted, the calculations employed the aug-cc-pVDZ basis set [41]. The orbitals were obtained in tightly converged CASSCF calculations using the reference spaces listed in Table 1. All configurations in the CAS space including those of symmetry different from the ground state were used as reference configurations in the following uncontracted MRCI calculations. All unique single- and double-excitations relative to all reference configurations were included in the variational calculation without selection. Core electrons were not correlated. The linear equation system for the perturbed MRCI coefficients were solved by Pople's algorithm [42] and usually converged in less than 10 iterations to seven digits accuracy. The origin was chosen at the centre of the MRCI electronic charge. 


\section{Results and Discussion}

In general, the MRCI energies in Table 1 are 1-3 mEh higher than the corresponding numbers from spin-unrestricted coupled-cluster calculations with single- and double-excitations together with a perturbative triple-excitations correction $(\operatorname{CCSD}(\mathrm{T}))$. If it is assumed that the $\operatorname{CCSD}(\mathrm{T})$ results are close to full CI values, the main source of this discrepancy is very likely the size consistency error of the MRCI method. It would be remedied by the multireference Davidson correction. However, since the response of the (nonvariational) Davidson correction [43] has not been coded this would not have affected the outcome of the property calculations. Basis set convergence and core-correlation. Since the basis set convergence of the g-tensor has never been systematically studied at a correlated $a b$ initio level, the g-tensor of the $\mathrm{NH}$ molecule was investigated with the converging series of correlation consistent basis sets. As becomes evident from Table 2, the first order terms are easily converged to about $1 \mathrm{ppm}$ already at the triple- $\zeta$ level. This is, however, not true for the second order PSO term. Here, convergence is as slow as for the dynamic correlation energy itself and even upon going from cc-PVQZ to cc-pV5Z changes on the order of $20 \mathrm{ppm}$ are observed. The data in Table 2 fit reasonably well to a $\mathrm{X}^{-3}$ dependence of the $g_{\perp}^{(P S O)}$ shift with $\mathrm{X}$ being the cardinal number of the basis set. This fit leads to a predicted basis set limit of $\sim 1610 \mathrm{ppm}$ for the $g_{\perp}^{(P S O)}$-value of $\mathrm{NH}$ at the unrelaxed LRT-MRCI level in conjunction with the effective nuclear charge SOC operator. The effects of core-correlation were also briefly investigated for the $\mathrm{NH}$ molecule. Using the aug-pCVDZ basis set, $g_{\perp}^{(P S O)}$ is $1447.9 \mathrm{ppm}$ if only the valence electrons are correlated and $1479.5 \mathrm{ppm}$ upon including the $\mathrm{N}-1 \mathrm{~s}$ orbital in the correlation treatment. Thus, at least in this example, the core-correlation effects appear to be limited and will only play an important role upon approaching the basis set limit. The core-correlation effects are also not expected to be large since, unlike the case met in the calculation of the Fermi-contact interaction, the limited core-level spin-polarization is not expected to play an important role 
for the spin-orbit coupling of the valence shell electrons. Nevertheless, this subject certainly warrants a much more careful investigation on a larger test set of molecules including also heavier elements.

Comparison to experiment and other calculations. Comparison of the present calculations to experimental data is of limited value since small basis sets are used here and the experimental data are uncertain and often significantly affected by matrix effects. Hence, comparison can be made to previous SOS calculations of Brownridge et al.,[16] Bolvin [23] and the present author.[18] However, all of the previous calculations used larger basis sets and introduced additional approximations leading to additional uncertainties. Nevertheless, the results of the LRT-MRCI calculations do agree reasonably well with the previous SOS-MRCI calculations except for $\mathrm{H}_{2} \mathrm{O}^{+}$and $\mathrm{AlO}$. The latter case is particularly pathological and has recently been analyzed in much more detail by Gilka and Marian.[44] In this work, the LRT-MRCI calculations for $\mathrm{AlO}$ give reasonable results that appear to be stable.

For $\mathrm{H}_{2} \mathrm{O}^{+}$the results of the LRT-MRCI calculations seem to be inferior to the earlier SOSMRCI calculations (the $\mathrm{g}_{\max }$ shift is predicted too low in the present calculations).[12,16,18,23] The problems appear to be associated with the CASSCF orbitals as will be discussed in more detail below.

Spin orbit operators. It is evident from Table 3 - Table 5 that the effective nuclear charge and SOMF SOC Hamiltonians provide results within $\sim 10 \%$ of each other. The motivation for including the less rigorous effective nuclear charge values is that this operator is easily implemented and this will facilitate future comparison of theoretical g-tensor results. Since the effective nuclear charge values always overshoot the more rigorous SOMF results, it would have been possible to improve on the effective nuclear charge model by dividing the effective charges by $\sim 1.1-1.2$. However, this would be a merely cosmetic operation. Discussion of Approximations. In obtaining the theoretical results of this work only two approximations above the limitations of a finite (small) one-particle have been made: (1) 
neglect of the dependence of the basis set on the (magnetic field) perturbation and (2) neglect of the orbital relaxation contribution. The first approximation is not very severe as all calculations to date show that the gauge dependence of the g-tensor is small. Hence, the enormous effort to implement gauge-including atomic orbitals (GIAO's) into the LRT-MRCI code does not seem worthwhile at this stage. Concerning the second approximation, it is obvious that the orbital relaxation contributions must vanish for a full CI wavefunction.[28] To the extent that one hopes to approximate this limit well in the present calculations it could also be hoped that the orbital relaxation contributions are small enough to be neglected. Indeed, in CC calculations of response properties the orbital response is often neglected in order to avoid spurious poles that are introduced via the Hartree-Fock ground state (see for example the interesting discussion in ref [45]). Orbital relaxation contributions are implicitly accounted for in such calculations through the higher powers of the $t_{1}$ (single-excitation cluster) operator. A similar situation does, however, not exist in the case of CI calculations which are linear in the single excitation amplitudes. Thus, if the orbitals are optimized for the ground state, it might happen that the excited states are not well described by the same orbitals and consequently, one might expect that the linear response treatment would predict g-shifts that are too small in magnitude.

In order to find out how large these effects are in the case of g-tensors, calculations with different sets of orbitals were carried out for $\mathrm{NH}, \mathrm{CN}$ and $\mathrm{H}_{2} \mathrm{O}^{+}$. For this purpose it is sufficient to focus on the largest PSO contribution to the g-shift. For NH the largest contribution to the $g_{\perp}^{(P S O)}$-shift in a SOS picture arises from the low-lying ${ }^{3} \Pi$ state. Thus, it seems appropriate to compare calculations where the reference orbitals have been obtained either from single root $\operatorname{CASSCF}(6,5)$ and state-averaged (SA) three-root $\operatorname{CASSCF}(6,5)$ calculations. The resulting $g_{\perp}^{(P S O)}$ values are $1418.9 \mathrm{ppm}$ and $1461.3 \mathrm{ppm}$ which amounts to a change of less than 3\%. Similarly, for the CN-radical it was shown previously, that the largest 
contribution to $g_{\perp}^{(P S O)}$ does not arise from the low-lying $1-^{2} \Pi$ state but from the much higher $2-{ }^{2} \Pi$ state since couples much more strongly to the ground state than $1-{ }^{2} \Pi .[18]$ In fact, orbitals from a $\operatorname{CASSCF}(9,8)$ yield $g_{\perp}^{(P S O)}=-1889 \mathrm{ppm}$ and a seven root average, which includes the $2-^{2} \Pi$ state yields $g_{\perp}^{(P S O)}=-1882 \mathrm{ppm}$. This result indicates that orbital relaxation effects are also limited in this system.

However, one should not jump to the conclusion that the dependence of the results on the orbitals is always small. In fact, this is not the case - turning to $\mathrm{H}_{2} \mathrm{O}^{+}$, the largest contribution to $g_{33}^{(P S O)}$ arises from the low-lying $1-^{2} \mathrm{~A}_{1}$ state that is experimentally found around 16,500 $\mathrm{cm}^{-1}$.[12] In this system orbital averaging makes a very large difference. Determining the orbitals from a single root $\operatorname{CASSCF}(7,6)$ calculation yields a LRT-MRCI value $g_{33}^{(P S O)}=12596$ ppm while two root averaging gives $g_{33}^{(P S O)}=15482 \mathrm{ppm}-$ a change as large as $20 \%$. The seemingly better result of the two-root calculation (the experimental value for the $g_{33}^{(t o t)}$ shift is $\sim 18800 \mathrm{ppm}$ [12]) may well be associated with the averaged orbitals that describe the crucial ${ }^{2} \mathrm{~B}_{1} \rightarrow{ }^{2} \mathrm{~A}_{1}$ excitation in a more balanced way than the single root orbitals. In fact, already the SA-CASSCF transition energy of the two-root calculation $\left(18500 \mathrm{~cm}^{-1}\right)$ is close to experiment while the orbitals of the single-root optimization yield $24900 \mathrm{~cm}^{-1}$ which is much too high. This shortcoming is not repaired at the MRCI level, where the transition energy is still predicted too high with the single root orbitals $\left(20400 \mathrm{~cm}^{-1}\right)$ while the corresponding value with the two-root orbitals is accurate $\left(16400 \mathrm{~cm}^{-1}\right)$. This shows once more that it is important for linear response treatments of ground state properties that the eigenvalues of the response matrix (for LRT-MRCI simply $\mathbf{H}^{(0)}-\mathbf{1} E_{M R C I}$ ) describe excitation energies well. It is noted in passing that the MRCI calculation based on the two-root CASSCF orbitals even yield a MRCI energy that is slightly lower $(0.2 \mathrm{mEh})$ than the MRCI solution based on the singleroot CASSCF orbitals. Whether a LRT-MRCI calculation that includes orbital relaxation is 
able to compensate for the shortcomings of the single-root CASSCF orbitals appears to be an open question. It is noted, however, that the significant deviations from experiment that still persist are not due to basis set effects. Calculations with the cc-pVQZ basis and the SOMF operator still yield a $g_{33}^{(P S O)}$ of only $\sim 15616 \mathrm{ppm}$ which is $\sim 20 \%$ below the experimental gasphase value.

\section{Conclusions}

In the present work the first analytic derivative implementation of electronic g-tensors at the MRCI level is reported and has been evaluated through benchmark calculations on small molecules. For a given set of molecular orbitals, the present LRT-MRCI approach is to be preferred over SOS based treatments since the entire manifold of excited states is implicitly accounted for and no truncation error arises. Under the reasonable assumption that the solution of the CP-MRCI equations is computationally about as expensive as the calculation of a single MRCI root, the LRT-MRCI method is also computationally more economical than the SOS-MRCI treatment.

However, the present implementation is not complete in the sense that the orbital relaxation contributions are neglected. While it was initially hoped that the MRCI wavefunction might be accurate enough in order to compensate for this neglect, the actual calculations suggest that this is probably at least not always the case - different sets of state averaged CASSCF orbitals have yielded very different response contributions to the g-tensor for $\mathrm{H}_{2} \mathrm{O}^{+}$. In situations with low lying excited states that strongly spin-orbit couple to the electronic ground state it may prove advantageous to determine the orbitals for an average of several low-lying states since this will allow for a more balanced treatment of excitation energies at the linear response level. For future high-accuracy studies it appears desirable to include the orbital response in such calculations. This requires the solution of the coupled-perturbed CASSCF equations and their derivatives for a purely imaginary perturbation. The development of an efficient 
computer code for this task is a significant undertaking that has so far not been completed in the framework of the ORCA program.

The main fields of application for such an involved methodology might be: (a) accurate calculations on small molecules (owing to the slow basis set convergence this will likely also require basis set extrapolation to be carried out), (b) generation of benchmark values for more approximate schemes and (c) application to molecules with genuine multireference character. The lack of size consistency is a serious issue in such calculations and might be dealt with through average coupled pair functionals.[46] Once these tasks are accomplished, additional approximations must be introduced in order to make LRT-MRCI g-tensor calculations computationally feasible for at least medium sized molecules. Work in this direction is in progress in our group.

Acknowledgement. It is my pleasure to dedicate this paper to Prof. Peter Pulay in recognition of his outstanding contributions to quantum chemistry. I also gratefully acknowledge financial support from the german science foundation (DFG priority program 1137, SFB 624 and SFB 663), the German-Israeli foundation and the university of Bonn. 
Table 1: Geometries (in a.u. and degrees), CASSCF energies, MRCI energies and CCSD(T) energies (all in Eh)obtained for the test set in the aug-cc-pVDZ basis.

\begin{tabular}{|l|c|r|r|l|l|}
\hline Molecule & $\mathrm{E}(\mathrm{CASSCF})$ & $\mathrm{E}(\mathrm{MR}-\mathrm{CI})$ & $\mathrm{E}(\mathrm{CCSD}(\mathrm{T}))$ & Ref-Space & Geometry \\
\hline $\mathrm{CN}$ & -92.351608 & -92.501359 & -92.502281 & $\mathrm{CAS}(9,8)$ & $\mathrm{R}_{\mathrm{CN}}=2.2140$ \\
\hline $\mathrm{CO}^{+}$ & -112.423319 & -112.564973 & -112.565419 & $\mathrm{CAS}(9,8)$ & $\mathrm{R}_{\mathrm{CO}}=2.0986$ \\
\hline $\mathrm{BO}$ & -99.651369 & -99.793545 & -99.796694 & $\mathrm{CAS}(9,8)$ & $\mathrm{R}_{\mathrm{BO}}=2.2770$ \\
\hline $\mathrm{AlO}$ & -316.863176 & -317.010044 & -317.011757 & $\mathrm{CAS}(9,8)$ & $\mathrm{R}_{\mathrm{AlO}}=3.0569$ \\
\hline $\mathrm{NH}$ & -54.990463 & -55.103281 & -55.105587 & $\mathrm{CAS}(6,5)$ & $\mathrm{R}_{\mathrm{NH}}=1.9578$ \\
\hline $\mathrm{OH}^{+}$ & -75.005612 & -75.116515 & -75.117897 & $\mathrm{CAS}(6,5)$ & $\mathrm{R}_{\mathrm{OH}}=1.9446$ \\
\hline $\mathrm{PH}$ & -341.301599 & -341.397667 & -341.400433 & $\mathrm{CAS}(6,5)$ & $\mathrm{R}_{\mathrm{PH}}=2.6736$ \\
\hline $\mathrm{SH}^{+}$ & -397.770501 & -397.868444 & -397.870574 & $\mathrm{CAS}(6,5)$ & $\mathrm{R}_{\mathrm{SH}}=2.5965$ \\
\hline $\mathrm{H}_{2} \mathrm{O}^{+}$ & -75.677504 & -75.803890 & -75.806194 & $\mathrm{CAS}(7,6)$ & $\mathrm{R}_{\mathrm{OH}}=2.0893 \angle 109.62$ \\
\hline $\mathrm{NH}_{2}$ & -55.618776 & -55.747935 & -55.751555 & $\mathrm{CAS}(7,6)$ & $\mathrm{R}_{\mathrm{NH}}=1.9291 \angle 103.15$ \\
\hline $\mathrm{H}_{2} \mathrm{~S}^{+}$ & -398.392575 & -398.504049 & -398.506884 & $\mathrm{CAS}(7,6)$ & $\mathrm{R}_{\mathrm{SH}}=2.5614 \angle 93.30$ \\
\hline $\mathrm{PH}_{2}$ & -341.904247 & -342.013942 & -342.017597 & $\mathrm{CAS}(7,6)$ & $\mathrm{R}_{\mathrm{PH}}=2.6663 \angle 91.92$ \\
\hline
\end{tabular}

Table 2: Basis set convergence of LRT-MRCI g-tensor (in ppm) calculations for $\mathrm{NH}\left({ }^{3} \Sigma\right)$ (effective nuclear charge $\mathrm{SOC}$ operator)

\begin{tabular}{|l|l|l|l|l|l|l|}
\hline Basis Set & $g_{\|}^{(t o t)}$ & $g_{\perp}^{(t o t) \mathrm{a}}$ & $g^{(R M C)}$ & $g_{\|}^{(D S O)}$ & $g_{\perp}^{(D S O)}$ & $g_{\perp}^{(P S O) \mathrm{a}}$ \\
\hline cc-pVDZ & -107.4 & 1205.4 & -205.1 & 97.6 & 71.0 & 1339.5 \\
\hline cc-pVTZ & -107.8 & 1394.4 & -205.8 & 98.0 & 70.9 & 1529.2 \\
\hline cc-pVQZ & -107.8 & 1441.6 & -205.8 & 98.0 & 70.9 & 1576.6 \\
\hline cc-pV5Z & -107.8 & 1463.0 & -205.7 & 97.9 & 70.9 & 1597.8 \\
\hline
\end{tabular}

a $\mathrm{h}$ functions had to be deleted due to technical constraints. 
Table 3: MRCI linear response g-tensor (in ppm) results for ${ }^{2} \Sigma$ molecules as described in the text (results obtained with the effective nuclear charge SOC operator. Results obtained with the full SOMF-SOC operator are given in parenthesis).

\begin{tabular}{|l|l|l|l|l|l|l|}
\hline Molecule & $g_{\|}^{(\text {tot })}$ & $g_{\perp}^{(\text {tot }) \mathrm{a}}$ & $g^{(R M C)}$ & $g_{\|}^{(D S O)}$ & $g_{\perp}^{(D S O)}$ & $g_{\perp}^{(P S O) \mathrm{a}}$ \\
\hline $\mathrm{CN}$ & -125.7 & $-1946.8(-1758.8)$ & -170.5 & 44.8 & 113.5 & $-1889.8(-1701.8)$ \\
\hline $\mathrm{CO}^{+}$ & -125.4 & $-2361.2(-2144.9)$ & -173.5 & 48.1 & 122.1 & $-2309.8(-2093.6)$ \\
\hline $\mathrm{BO}$ & -61.3 & $-1664.9(-1493.5)$ & -90.8 & 29.5 & 78.8 & $-1652.9(-1481.5)$ \\
\hline $\mathrm{AlO}$ & -78.1 & $-2141.0(-1739.7)$ & -158.7 & 80.6 & 180.4 & $-2162.7(-1761.4)$ \\
\hline
\end{tabular}

Table 4: MRCI linear response g-tensor (in ppm) results for ${ }^{3} \Sigma$ molecules as described in the text (results obtained with the effective nuclear charge SOC operator. Results obtained with the full SOMF-SOC operator are given in parenthesis).

\begin{tabular}{|l|l|l|l|l|l|l|}
\hline Molecule & $g_{\|}^{(\text {tot })}$ & $g_{\perp}^{(\text {tot } \mathrm{a}}$ & $g^{(R M C)}$ & $g_{\|}^{(D S O)}$ & $g_{\perp}^{(D S O)}$ & $g_{\perp}^{(P S O) \text { a }}$ \\
\hline $\mathrm{NH}$ & -106.0 & $1286.6(1131.0)$ & -203.0 & 97.0 & 70.6 & $1418.9(1263.4)$ \\
\hline $\mathrm{OH}^{+}$ & -175.5 & $3619.9(3260.9)$ & -323.7 & 148.2 & 109.8 & $3822.8(3474.8)$ \\
\hline $\mathrm{PH}$ & -17.8 & $4201.5(3823.2)$ & -146.0 & 163.7 & 117.1 & $4230.4(3852.1)$ \\
\hline $\mathrm{SH}^{+}$ & -19.1 & $8068.6(7609.6)$ & -229.8 & 210.8 & 152.6 & $8145.9(7686.9)$ \\
\hline
\end{tabular}


Table 5: MRCI linear response g-tensor (in ppm) results for ${ }^{2} \mathrm{XH}_{2}{ }^{\mathrm{n+}}(\mathrm{n}=0,1)$ molecules with ${ }^{2} \mathrm{~B}_{1}$ ground states as described in the text (results obtained with the effective nuclear charge SOC operator. Results obtained with the full SOMF-SOC operator are given below).

\begin{tabular}{|l|l|l|l|l|l|l|l|l|l|l|}
\hline Molecule & $g_{11}^{(\text {tot })}$ & $g_{22}^{(\text {tot })}$ & $g_{33}^{(\text {tot })}$ & $g^{(\text {RMC })}$ & $g_{11}^{(\text {DSO })}$ & $g_{22}^{(\text {DSO })}$ & $g_{33}^{(\text {DSO })}$ & $g_{11}^{(\text {PSO })}$ & $g_{22}^{(P S O)}$ & $g_{33}^{(\text {PSO })}$ \\
\hline $\mathrm{H}_{2} \mathrm{O}^{+}$ & -191.2 & 4566.3 & 12427.1 & -317.5 & 73.2 & 146.7 & 147.9 & 53.1 & 4737.1 & 12598.3 \\
\hline & -198.1 & 4006.9 & 11117.0 & & & & & 46.3 & 4177.7 & 11286.7 \\
\hline $\mathrm{NH}_{2}$ & -144.9 & 1532.3 & 4767.1 & -198.3 & 45.9 & 97.1 & 98.6 & 7.5 & 1633.5 & 4866.9 \\
\hline & -147.9 & 1312.7 & 4183.9 & & & & & 4.6 & 1414.0 & 4283.6 \\
\hline $\mathrm{H}_{2} \mathrm{~S}^{+}$ & 41.3 & 10698.9 & 24088.9 & -228.7 & 97.6 & 212.0 & 210.3 & 172.4 & 10715.6 & 24107.3 \\
\hline & 24.0 & 10071.1 & 22727.0 & & & & & 155.1 & 10087.8 & 22745.4 \\
\hline $\mathrm{PH}_{2}$ & 24.6 & 4707.4 & 13622.3 & -146.1 & 74.1 & 165.6 & 164.0 & 96.6 & 4687.9 & 13604.5 \\
\hline & 8.9 & 4272.5 & 12409.6 & & & & & 80.9 & 4252.9 & 12391.8 \\
\hline
\end{tabular}




\section{Literature}

[1] F. Neese, in: B. Gilbert (Ed.), Specialist Reports on EPR Spectroscopy. Royal Society of Chemistry, London, 2007, p. 73.

[2] F. Neese Biol. Mag. Res. (Ed. G. Hanson) (2007) in press.

[3] S. Patchkovskii, G. Schreckenbach, in: M. Kaupp, V. Malkin, M. Bühl (Eds.), Calculation of NMR and EPR Parameters. Wiley-VCH, Weinheim, 2004, p. 505.

[4] G. Schreckenbach, T. Ziegler J. Phys. Chem. A. 101 (1997) 3388.

[5] F. Neese J. Chem. Phys. 115 (2001) 11080.

[6] D. Jayatilaka J. Chem. Phys 108 (1998) 7587.

[7] P. Bündgen, G. H. Lushington, F. Grein Int. J. Quant. Chem. 29 (1995) 283.

[8] G. H. Lushington, P. Bündgen, F. Grein Int. J. Quant. Chem. 55 (1995) 377.

[9] P. J. Bruna, G. H. Lushington, F. Grein Chem. Phys. Lett. 258 (1996) 427.

[10] G. H. Lushington, F. Grein Theor. Chim. Acta 93 (1996) 259.

[11] G. H. Lushington, F. Grein Int. J. Quant. Chem.: Quant. Chem. Symp. 30 (1996) 467.

[12] G. H. Lushington, F. Grein J. Chem. Phys. 106 (1997) 3292.

[13] P. J. Bruna, F. Grein J. Chem. Phys. 109 (1998) 9439.

[14] P. J. Bruna, F. Grein Int. J. Quant. Chem 77 (2000) 324.

[15] P. J. Bruna, F. Grein Chem. Phys. Lett. 318 (2000) 263.

[16] S. Brownridge, F. Grein, J. Tatchen, M. Kleinschmidt, C. M. Marian J. Chem. Phys. 118 (2003) 9552.

[17] F. Neese Mag. Res. Chem. 42 (2004) S187.

[18] F. Neese Chem. Phys. Lett. 380 (2003) 721.

[19] F. Neese Int. J. Quant. Chem. 83 (2001) 104.

[20] A. Delabie, K. Pierloot, M. H. Groothaert, B. M. Weckhuysen, R. A. Schoonheydt Phys. Chem. Chem. Phys. 4 (2002) 134.

[21] K. Pierloot, A. Delabie, M. H. Groothaert, R. A. Schoonheydt Phys. Chem. Chem. Phys. 3 (2001) 2174.

[22] H. Bolvin Inorg. Chem. 46 (2007) 417

[23] H. Bolvin Chem. Phys. Chem. 7 (2006) 1575.

[24] O. Vahtras, M. Engström, B. Schimmelpfennig Chem. Phys. Lett. 351 (2002) 424.

[25] M. Engström, O. Vahtras, H. Ågren Chem. Phys. Lett. 243 (1999) 263.

[26] M. Engström, B. Minaev, O. Vahtras, H. Agren Chem. Phys. Lett. 237 (1998) 149.

[27] O. Vahtras, B. Minaev, H. Agren Chem. Phys. Lett. 281 (1997) 186.

[28] M. Kallay, J. Gauss J. Chem. Phys. 120 (2004) 6841.

[29] M. Kallay, J. Gauss, P. G. Szalay J. Chem. Phys. 119 (2003) 2991.

[30] R. McWeeny: Methods of Molecular Quantum Mechanics, Academic press, London, 1992.

[31] H. Lischka, R. Shepard, R. M. Pitzer, I. Shavitt, M. Dallos, T. Muller, P. G. Szalay, M. Seth, G. S. Kedziora, S. Yabushita, Z. Y. Zhang Phys. Chem. Chem. Phys. 3 (2001) 664.

[32] D. Ganyushin, F. Neese J. Chem. Phys. 125 (2006) 024103.

[33] S. Koseki, M. W. Schmidt, M. S. Gordon J. Chem. Phys. 96 (1992) 10768.

[34] S. Koseki, M. S. Gordon, M. W. Schmidt, N. Matsunaga J. Phys. Chem. 99 (1995) 12764.

[35] S. Koseki, M. W. Schmidt, M. S. Gordon J. Phys. Chem. A. 102 (1998) 10430.

| [36] B. A. Hess, C. M. Marian, U. Wahlgren, O. Gropen Chem. Phys. Lett. 251 (1996) 365.

[37] B. Schimmelpfennig AMFI - an atomic mean field integral program. University of Stockholm, Stockholm, Sweden, 1996.

[38] F. Neese J. Chem. Phys. 122 (2005) 034107/1. 
[39] A. Berning, M. Schweizer, H. J. Werner, P. J. Knowles, P. Palmieri Molec. Phys. 98 (2000) 1823.

[40] F. Neese ORCA - an ab initio, density functional and semiempirical program package, University of Bonn, Germany, 2007.

[41] J. T. H. Dunning J. Chem. Phys. 90 (1989) 1007.

[42] J. A. Pople, R. Krishnan, H. B. Schlegel, J. S. Binkley Int. J. Quant. Chem.: Quant. Chem. Symp. 13 (1979) 225.

[43] G. Hirsch, P. J. Bruna, S. D. Peyerimhoff, R. J. Buenker Chem. Phys. Lett. 52 (1977) 442.

[44] N. Gilka, C. M. Marian Chem. Phys. (2007) submitted for publication.

[45] P. G. Szalay, J. Vazquez, C. Simmons, J. F. Stanton J. Chem. Phys. 121 (2004) 7624.

[46] R. J. Gdanitz, R. Ahlrichs Chem. Phys. Lett. 143 (1988) 413. 